\title{
A STRAIN-BASED PLASTIC VISCOUS-DAMAGE MODEL FOR MASSIVE CONCRETE STRUCTURES
}

\author{
Rui FARIA \\ Faculdade de Engenharia, Universidade do Porto, Rua dos Bragas, 4099 Porto Codex, Portugal

\section{JAVIER OLIVER and Miguel CERVERA} \\ E.T.S. Ingenieros de Caminos, Canales y Puertos, Technical University of Catalonia \\ C/ Gran Capitán s/n, Edificio C1, 08034 Barcelona, Spain
}

\begin{abstract}
Within the framework of Continuum Damage Mechanics, a new constitutive damage model for massive concrete is presented, mainly intended for the seismic analysis of gravity and arch dams. Consistent with thermodynamic requirements, a strain-driven formalism is adopted, improving the algorithmic efficiency as much as required for the analysis of large scale problems to become feasible. Two scalar damage variables are introduced as internal variables, as well as a plastic-strain tensor. Extension to account for the concrete strain-rate dependency, suitable for seismic analysis, is presented at the end. Efficiency of numerical predictions from the constitutive model is illustrated through numerical applications. Algorithmic implementation is also detailed.
\end{abstract}

\section{INTRODUCTION}

Among the wide variety of problems to be solved in Structural Engineering, the analysis of concrete massive structures (like dams) is widely recognised as rather peculiar, mainly due to the large scale problem resulting from an appropriate volumetric discretization, particularly if a seismic analysis is to be performed, where a refined time-stepping scheme is usually required.

Due to this 'scale' problem, the structural behaviour of concrete dams only rarely is ascertained taking into consideration its non-linear material constitutive behaviour, particularly if $3 \mathrm{D}$ analyses have to be considered [1]. Most commonly, non-linear analyses of concrete dams are performed in a posteriori fashion, for interpretation of pathologies or during scientific investigation, and only seldom for design purposes. Some 
attention must then be devoted to the appropriate selection of a constitutive model adequate for the seismic analysis of large scale concrete structures, so that its computational effort is kept within reasonable limits as much as possible, for the intended analysis to become feasible.

In Section 2 an original numerical model is presented, supported by a strain-based formalism which ensures high algorithmic efficiency. The following basic features of concrete behaviour were selected as relevant to be modelled, so that physically realistic predictions could be expected: $(i)$ the rather distinct stress-strain envelopes obtained under tension or under comprosion, with large differences in their peak strengths; $(i i)$

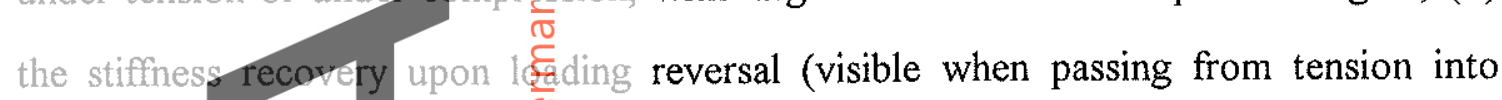
compression, or backvards); $\frac{ \pm}{3} i$ ) the concrete strength enhancement discernible under 2D or 3D compressive tests, weren compared to the $1 \mathrm{D}$ compressive strength and (iv) the plastic deformations observablë being attained. Two scalar da像age variables are introduced, each of them linked to the degradation mechanisms occe(assumed as independent). Aplepopriate evolution laws are cast for the damage variables, and also for a plastic strain terisor, added to the internal variables ensemble. Taking into consideration relevant thermodinamic principles, dissipation is checked and an intuitive constitutive law is derived. algorithmicimplementation, as well as the validation of the model predictions.

Section 3 is devoted to the modelling of the rate sensitivity exhibited by concrete

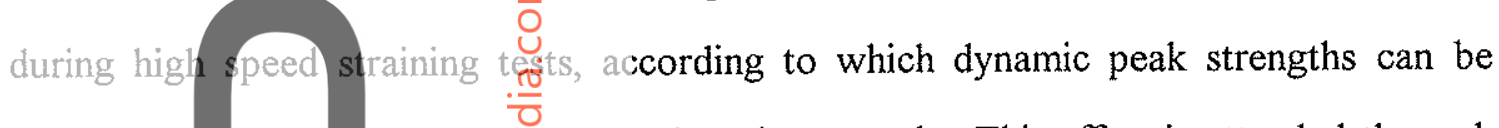
significantly enhanced reintivelg to quasi-static strengths. This effect is attended through a slight modification on the eqolution laws for the threshold variables involved in the constitutiv model described insection 2, conceptually similar to the viscoplastic Perzina regularization. The overall fhorithm for the inviscid constitutive model is kept unchanged, the only modificatron being the procedure for the updating of the damage thresholds. Some applicationd illustrate the good agreement between the model predictions and pertinent expe $\frac{4}{0}$ imental results. 


\section{RATE-INDEPENDENT PLASTIC-DAMAGE MODEL}

As widely recognised, Continuum Damage Mechanics provides a powerful and general framework, based on Thermodynamics of Irreversible Processes, from which the derivation of consistent material models is possible for many engineering fields. Firstly introduced by Kachanov [2] for creep-related problems, nowadays Damage Mechanics has a wide range of applicability, for materials so different as steel, ceramics, rock and concrete $[3-15]$

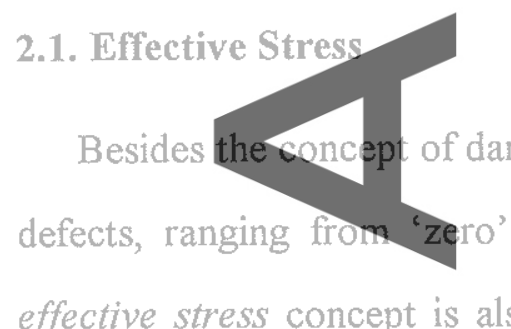

effective stress concept is also

With reference to the ID situation schematically depicted in Fig. 1, where $S$ denotes the overall cross sectional area and $\bar{S}$ the effective resistant section (the area $S-\bar{S}$ of the notch symbolises the area of the material defects), distinction between the usual Cauchy stress $\sigma$ and the effective stres $\bar{\sigma}$ is quite evident. A remark is made to the fact of $\bar{\sigma}$ being physically more represe load is effectively applied on $\frac{O}{\frac{O}{2}}$ and not on $S$.

Strictly connected to the effective stress concept, the hypothesis of strain equivalence is also introducec "The strain associated with a damage state under the applied stress $\sigma$ is equivalent $t \frac{\varepsilon}{\Phi}$ the strain associated with its undamaged state under the

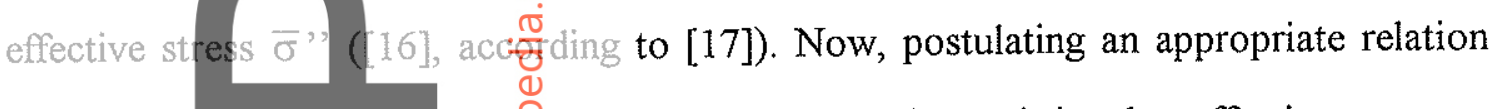
between stresses and strains $\frac{0}{0}$ for naterial points located in the effective area, a constitutive law can be derize in a more comprehensive way. Let us assume the following definition for $\bar{\sigma}$, a thee-dimensional effective stress tensor (rank two)

$$
\text { 产 }\left(\varepsilon, \varepsilon^{p}\right)=D_{0}:\left(\varepsilon-\varepsilon^{p}\right)
$$

where $D_{0}$ denotes the usual fairth order isotropic linear-elastic constitutive matrix, $\varepsilon$ is the strain tensor and $\varepsilon^{p}$ corres $\underset{0}{\stackrel{d}{ \pm}}$ ponds to a plastic strain tensor (both rank two). $\boldsymbol{\varepsilon}$ is an

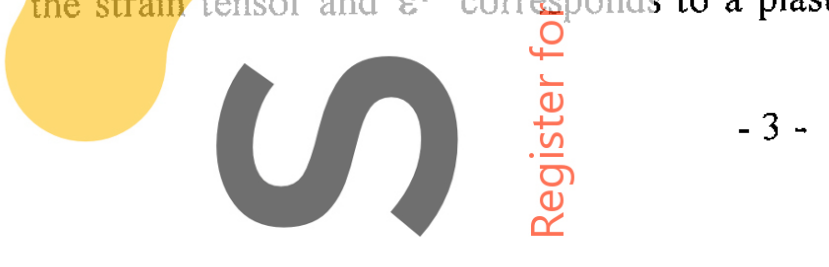


external (free) variable, whereas $\varepsilon^{p}$ is an internal one, with an evolution to be discussed latter.

In order to clearly distinguish stress contributions due to tension or to compression, thereafter assumed to produce independent non-linear mechanisms of degradation, a split of the effective stress tensor $\bar{\sigma}$ into tensile and compressive components $\left(\bar{\sigma}^{+}, \bar{\sigma}^{-}\right)$will be introduced, and performed according to

$$
\bar{\sigma}^{+}=\sum_{i}<\bar{\sigma}_{i}>p_{i} \otimes p_{i}
$$

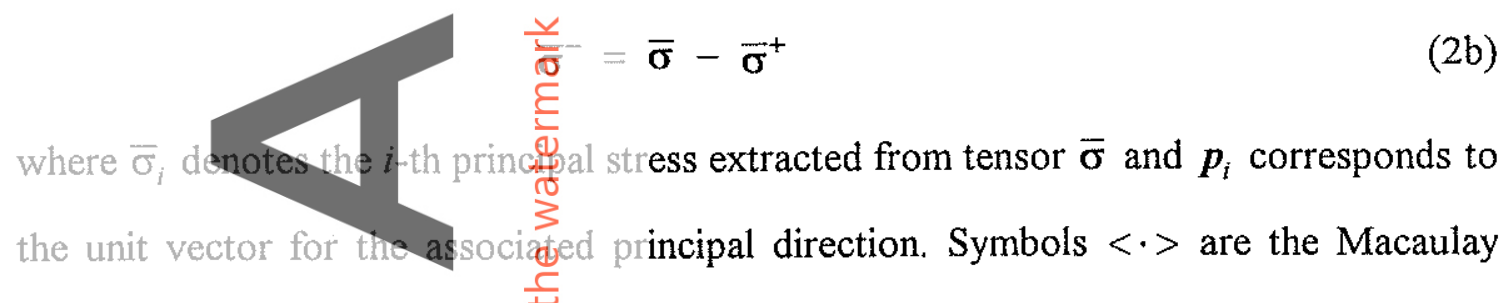
brackets (ramp function), thus and setting a zero value if negative; indices $(+)$ and $(-)$ will be extensively used hereafter, to point out tensile and compressive entities, respectively.

\subsection{Helmholtz Free Thergy Potential}

Continuum Damage Mectinics is based on the Thermodynamics of Irreversible Processes [18]. For a constitutite law to be established, a free energy potential must be introduced, in which the free $\frac{\text { Dnd }}{0}$ the internal variables have to be represented. Let us postulate a Helmholtz Free En\& $9 y$ Potential with the form [19]

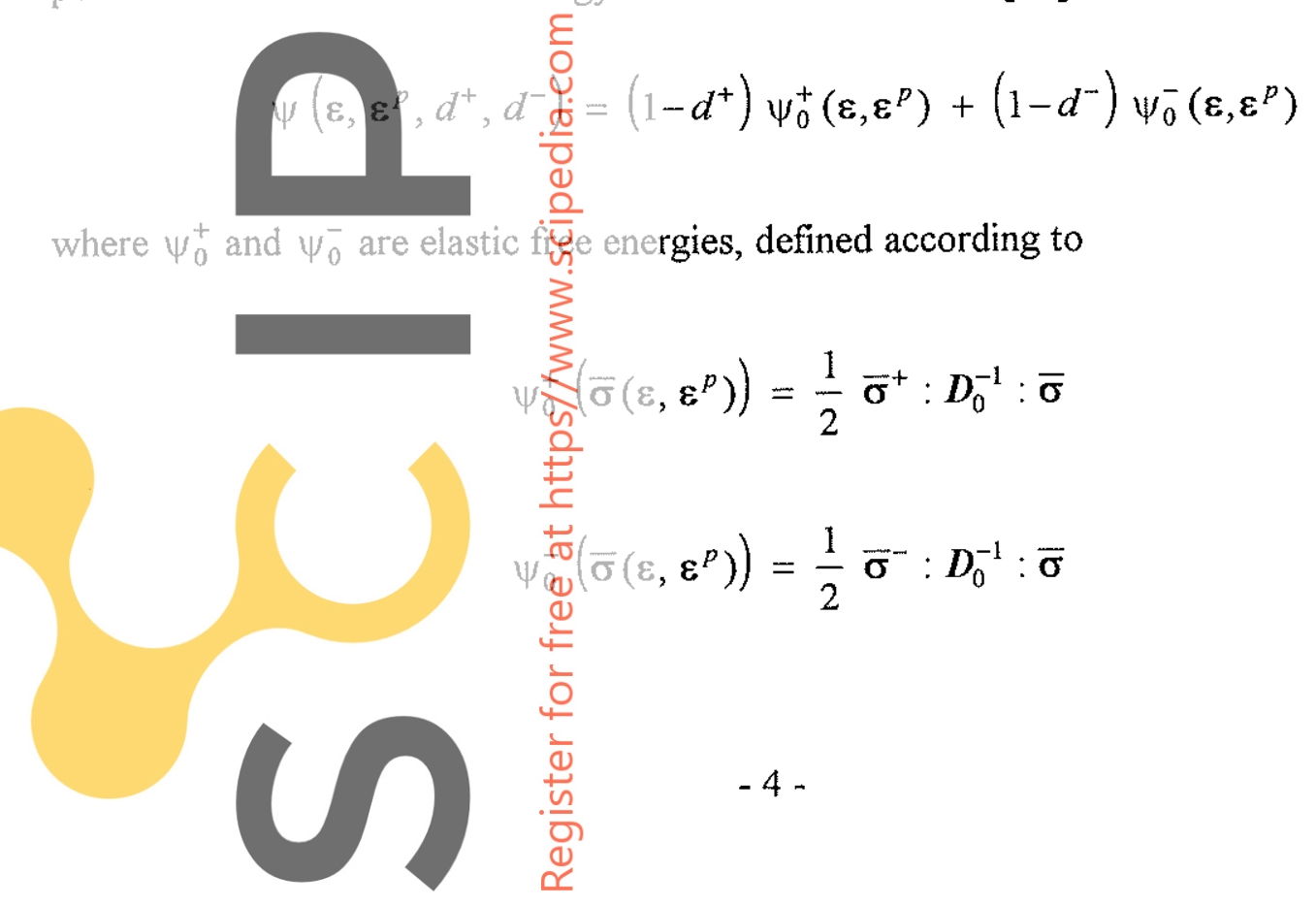


The internal variables set is constituted by the plastic strain tensor $\varepsilon^{p}$, as well as $d^{+}$and $d^{-}$, scalar damage variables ${ }^{\dagger}$ directly linked to tensile and compressive deteriorations, here assumed as corresponding to independent processes; strain tensor $\varepsilon$ is the single free variable admitted. $D_{0}^{-1}$ is the linear-elastic compliance matrix, which may assume the following definition, taking into consideration the usual notation of $E, v$ and $\delta$ for the Young's modulus, the Poisson's ratio and the 'Kronecker delta':

$$
D_{0}^{-1}=D_{0 k l}^{-1}=\frac{1}{E}\left[\frac{1+v}{2}\left(\delta_{i k} \delta_{j l}+\delta_{i l} \delta_{j k}\right)-v \delta_{i j} \delta_{k l}\right]
$$

Remark 2.1. In the absenc $\frac{y}{8}$ of damage and plasticity $\left(d^{+}=d^{-}=0, \varepsilon^{p}=\mathbf{0}\right)$, the free

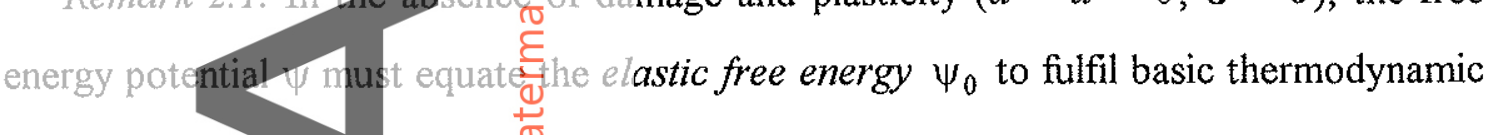
requirements, which according 30 eqns $(1-4)$ can be demonstrated to occur in the present situation:

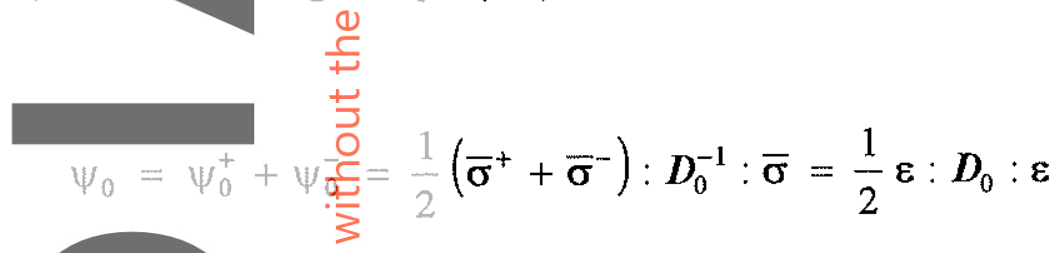

Note also that being $D_{0}$ a defiete and positive matrix it occurs that $\psi_{0} \geq 0$, due to the quadratic form of the last term $\frac{\frac{N}{Q}}{2}$ eqn (6).

Remark 2.2. The above de potentials proposed (or described) in [14-15]. Yet, in these references the free energies

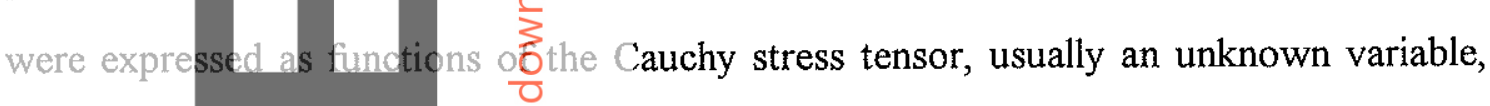
whilst in the present model eq $\$(3,4)$ only involve the effective stress tensor $\bar{\sigma}$, a rather more explicit entity, particular $\frac{\varepsilon}{\Phi}$ if $\varepsilon^{p}=\mathbf{0}$ (see eqn (1)).

In view of eqn (5), the defingtion of $\psi_{0}^{+}$given in eqn (4a) can be modified to render

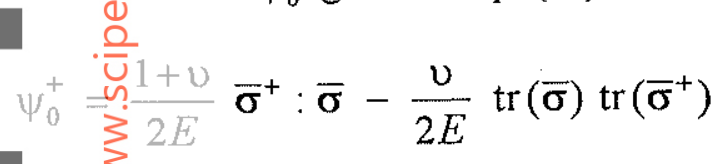

where $\operatorname{tr}(\cdot)$ is the trace of ten $(0)$. Taking into consideration the stress split described in eqns (2), owing to elementa $\hat{\beta}_{t}$ easonings the following properties apply:

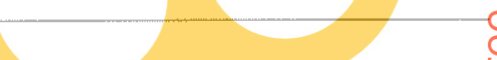

† Although tensor-valued damage tarrables [20-21] could also be adopted, a 'scalar' representation of damage is preferred, since it renderşess complicated algorithms, with sufficient approximation. 


$$
\begin{gathered}
\operatorname{tr}(\overline{\boldsymbol{\sigma}})=\operatorname{tr}\left(\overline{\boldsymbol{\sigma}}^{+}\right)+\operatorname{tr}\left(\overline{\boldsymbol{\sigma}}^{-}\right) \\
\bar{\sigma}^{+}: \overline{\boldsymbol{\sigma}}^{-}=0
\end{gathered}
$$

Back to eqn (7), it is now possible to express $\psi_{0}^{+}$as

$$
\begin{aligned}
\psi_{0}^{+} & =\frac{1+v}{2 E} \bar{\sigma}^{+}: \bar{\sigma}^{+}-\frac{v}{2 E} \operatorname{tr}^{2}\left(\overline{\boldsymbol{\sigma}}^{+}\right)-\frac{v}{2 E} \operatorname{tr}\left(\overline{\boldsymbol{\sigma}}^{--}\right) \operatorname{tr}\left(\overline{\boldsymbol{\sigma}}^{+}\right)= \\
& =\frac{1}{2} \overline{\boldsymbol{\sigma}}^{+}: \bar{D}_{0}^{-1}: \overline{\boldsymbol{\sigma}}^{+}+\left(-\frac{v}{2 E} \operatorname{tr}\left(\overline{\boldsymbol{\sigma}}^{-}\right) \operatorname{tr}\left(\overline{\boldsymbol{\sigma}}^{+}\right)\right)
\end{aligned}
$$

Due to the quadratic form of $1 / 2 \bar{\sigma}^{+}: \boldsymbol{D}_{0}^{-1}: \bar{\sigma}^{+}$, which involves the definite and positive $D_{0}^{-1}$ matrix, the first term in the right-hand side of eqn (9) is non-negative; the second term is also non-negative, $\sin c e \operatorname{tr}\left(\bar{\sigma}^{+}\right) \geq 0$ and $\operatorname{tr}\left(\bar{\sigma}^{-}\right) \leq 0$. According to these observations, it can then be conduded that

$$
\stackrel{3}{\stackrel{1}{c}} \quad \psi_{0}^{+} \geq 0
$$

By using a similar reasoning for $\psi_{0}^{-}$, it also applies that $\psi_{0}^{-} \geq 0$.

Owing to the non-negatives of $\psi_{0}^{+}$and $\psi_{0}^{-}$, and to the following requirements from the intrinsic damage variable concept

it can be put into evidence thatg

$$
0 \leq\left(d^{+}, d^{-}\right) \leq 1
$$

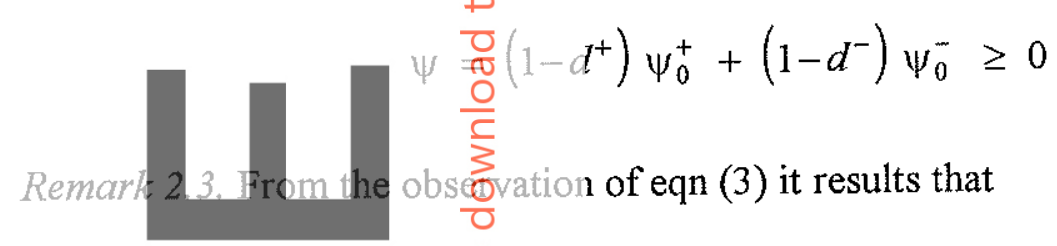

$$
-\frac{\partial \psi}{\partial d^{-}}=\psi_{0}^{-}
$$

where $\psi_{0}^{+}$and $\psi_{0}^{-}$can be loo $\frac{\mathrm{kg}}{\mathrm{d}} \mathrm{d}$ as the thermodynamic forces associated to the damage variables $d^{++}$and $d^{-}$, each of during an unit growth of the cospondent damage variable [7].

\subsection{Damage Criteria}

In order to clearly define scalar positive quantity, termed equivalent stress, will be introduced. Analogous to the

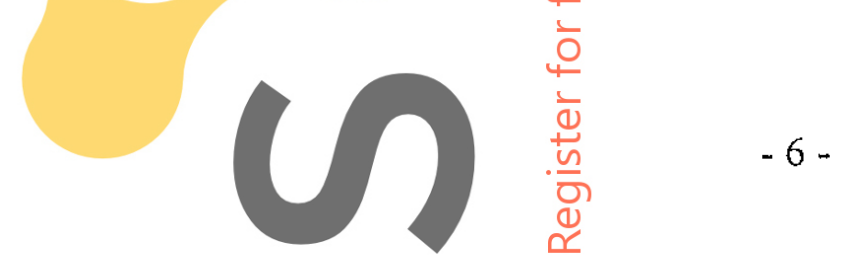


dual concept of equivalent strain established by Simo and $\mathrm{Ju}$ in [17], the equivalent stress provides a suitable norm for distinct 3D stress tensors, through which a mapping onto a single equivalent $1 \mathrm{D}$ stress test is then possible, thus enabling their quantitative comparison.

As a consequence of the stress split adopted throughout the present constitutive model, a tensile equivalent stress $\bar{\tau}^{+}$and a compressive equivalent stress $\bar{\tau}^{-}$will be considered. In the present work the following forms will be assumed $[17,19]$ :

$$
\begin{aligned}
& \bar{\tau}^{\bar{\tau}^{+}}=\sqrt{\bar{\sigma}^{+}: D_{0}^{-1}: \bar{\sigma}^{+}} \\
& \sum_{\frac{\delta}{\tau}}^{-}=\sqrt{\sqrt{3}\left(K \bar{\sigma}_{o c t}^{-}+\bar{\tau}_{\text {oct }}^{-}\right)}
\end{aligned}
$$

In the last equation $\sigma_{o x}$ and $\sum_{500}$ are the octahedral normal stress and the octahedral shear stress obtained from $\bar{\sigma} \stackrel{\oplus}{\Phi}$ is a material property, devised so that predicted $2 \mathrm{D}$ and ID compressive strengths coul $\overrightarrow{\vec{b}}$ match the usual 1.16-1.2 ratios reported for concrete in experimental tests [22]

With the al ready refered equinitions for the equalent stresses, and inspired in Ref. [17], two separated danage cristia $g^{+}$and $g^{-}$will be introduced, the former for tension and the latter for compression:

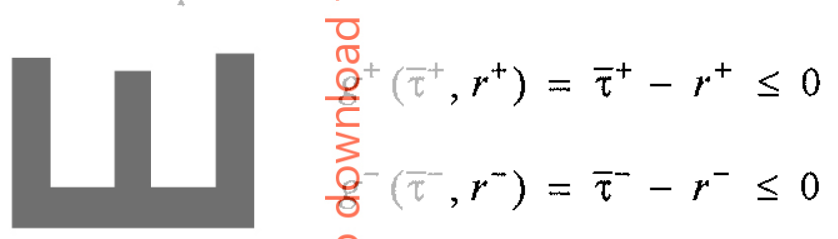

Variables $r^{+}$and $r^{-}$are curient damage thresholds, which control the size of the expanding danage sufaces. Açording to eqn (15a), previously to the application of any loading the $r^{+}$damage threshod must be set to $r_{0}^{+}$, assumed a material property, which bounds the linear-elastic domam. Following eqn (15b), a similar reasoning apply for compression, and therefore the nset of damage in compression will occur at $\bar{\tau}^{-}=r_{0}^{-}$.

From eqns (14a) and (15 in it results that within octant $\left(\bar{\sigma}_{1}, \bar{\sigma}_{2}, \bar{\sigma}_{3}\right) \geq 0$ the $3 \mathrm{D}$ effective stresses correspondin $\underset{8}{\vec{\sigma}}$ to the same norm $\bar{\tau}^{+}$define a quarter of an ellipsoid P 
centered at the origin in the space of principal effective stresses. Quadrant $\left(\bar{\sigma}_{1}, \bar{\sigma}_{3}\right) \geq 0$ in Fig. 2 provides a $2 \mathrm{D}$ representation for this surface, when $\bar{\sigma}_{2}=0$ and $\bar{\tau}^{+}=r_{0}^{+}$.

According to eqns (14b) and (15b), the bounding surface associated to the principal effective compressive stresses $\left(\left(\bar{\sigma}_{1}, \bar{\sigma}_{2}, \bar{\sigma}_{3}\right) \leq 0\right)$ resembles the Drucker-Pragger cone. As depicted in quadrant $\left(\bar{\sigma}_{1}, \bar{\sigma}_{3}\right) \leq 0\left(\bar{\sigma}_{2}=0\right)$ from Fig. 2, under 2D compression the elastic domain $\bar{\tau}^{-}=r_{0}^{-}$is bounded by stresses which are greater than the $1 \mathrm{D}$ elastic compressive stress $f_{0}^{-}$. Calibration of the model to reconstitute this phenomenon is performed through parameter $K$ in eqn (14b).

Fig. 2 shows also the exper mental results from Kupfer et al. [22], which are plotted against the numerical predictionfs from the present model, for comparison. The overall in tension-compression.

Remark 2.4. Defining as ${ }_{0}^{+}$and $f_{0}^{-}$the stresses beyond which non-linearity becomes visible under ID tesi (tension and compression, respectively), according to equs (14) the elastic thresholds $r_{0}^{+}$and $r_{0}^{-}$can be established as (note that for 1D tests $\sigma_{\text {oct }}=1 / 3 f$

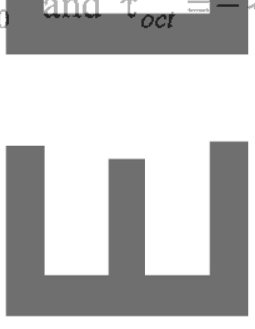

$$
\begin{aligned}
& \left(\begin{array}{c}
\left.3 f_{0}^{-}\right) \\
\hline
\end{array}\right. \\
& =\sqrt{f_{0}^{+} \frac{1}{E} f_{0}^{+}}=\frac{f_{0}^{+}}{\sqrt{E}} \\
& =\sqrt{\frac{\sqrt{3}}{3}(K-\sqrt{2}) f_{0}^{-}}
\end{aligned}
$$

Emination of parameter $K$ appearing in eqn (14b), information from two distinct $\frac{\mathbb{\overline { O }}}{\mathrm{Q}}$ ompressive tests is required. Let us consider a $1 \mathrm{D}$ test such that $\left(\sigma_{1}=0, \sigma_{2}=0, \sigma_{3} \cdot \frac{\rho}{\mathrm{j}^{\prime}} 0\right)$, and a $2 \mathrm{D}$ experiment where $\left(\sigma_{1}=0, \sigma_{2}=\sigma_{3} \leq 0\right)$, both driven until the maximumstress level compatible with linear-elasticity. Denoting by and $f_{02 \mathrm{D}}^{-}$the extreme valses obtained for $\sigma_{3}$ during those tests, it becomes evident that $\bar{\sigma}_{\text {oct } 1 \mathrm{D}}^{-}=1 / 3 f_{0}^{-}, \bar{\tau}_{\text {oct } 1 \mathrm{D}}^{-}=\stackrel{\stackrel{\rho}{ \pm}}{ \pm} \sqrt{2} / 3 f_{0}^{-}, \bar{\sigma}_{o c t 2 \mathrm{D}}^{-}=2 / 3 f_{02 \mathrm{D}}^{-}$and $\bar{\tau}_{\text {oct } 2 \mathrm{D}}^{-}=-\sqrt{2} / 3 f_{02 \mathrm{D}}^{-}$. Since both tests correspond $t \sigma_{\sigma}$ the same limit situation, the onset of non-linearity, they are characterised by the same eqtastic threshold $r_{0}^{-}$, which according to eqn (14b) leads to

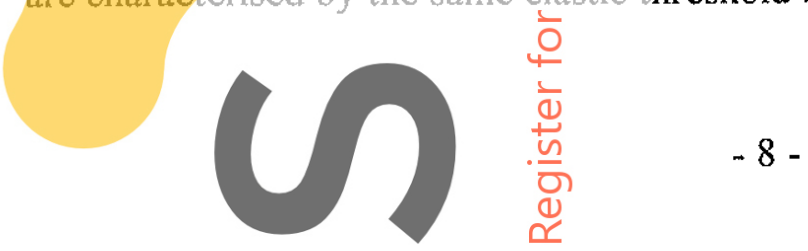




$$
K f_{0}^{-}-\sqrt{2} f_{0}^{-}=2 K f_{02 \mathrm{D}}^{-}-\sqrt{2} f_{02 \mathrm{D}}^{-}
$$

and consequently

$$
K=\sqrt{2} \frac{f_{02 \mathrm{D}}^{-}-f_{0}^{-}}{2 f_{02 \mathrm{D}}^{-}-f_{0}^{-}}
$$

\subsection{Evolution Laws for the Internal Variables}

\subsubsection{Damage variables}

For the kinematics of the damage variables the following rate equations will be assumed (exemplifying solely for tension):

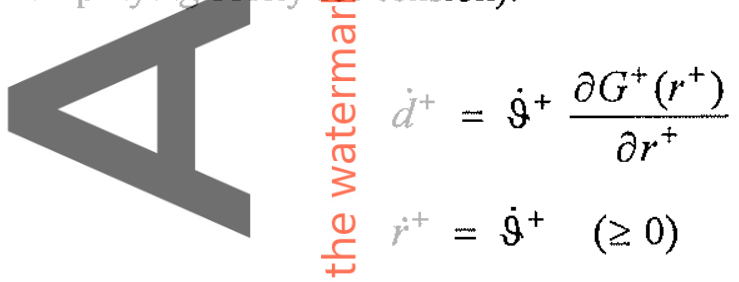

$G^{+}$, and dentically $G^{-}$for $\stackrel{+}{\sqsupset}$ compression, are monotonically increasing functions ${ }^{\dagger}$, selected in accordance with ${ }^{3}$ experimental observation. $\dot{\vartheta}^{+}$and $\dot{\vartheta}^{-}$are damage consistency parameters. relations:

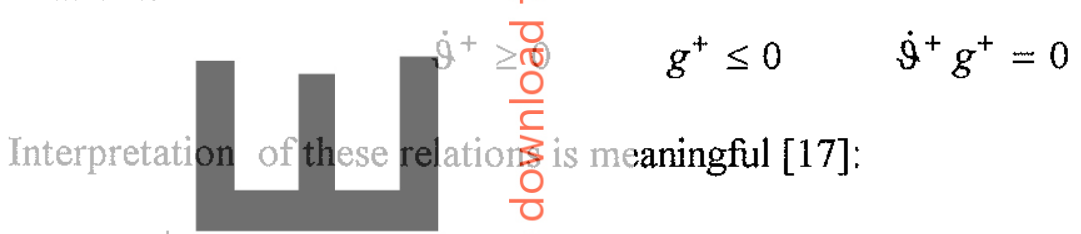

- $g^{+}<0$ states that no fipther damage is occurring, as it is clearly expressed by last equation in (20), impos $\underset{g}{E}$ that $\dot{\vartheta}^{+}=0$ (hence $\dot{d}^{+}=0$, owing to eqn (19a)).

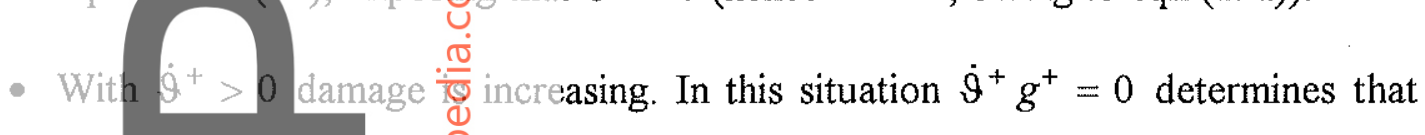
$g^{+}=0$, and so it is poside to define $\dot{\vartheta}^{+}$from the damage consistency condition:

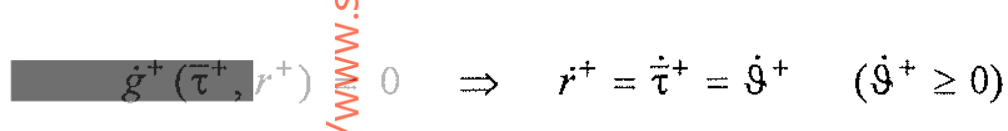

From the last condition it if

That is, first order derivatives $\partial G^{+} \underbrace{\frac{d}{2}}_{4} / \partial($.$) and \partial G^{-}(.) / \partial($.$) are non-negative.$

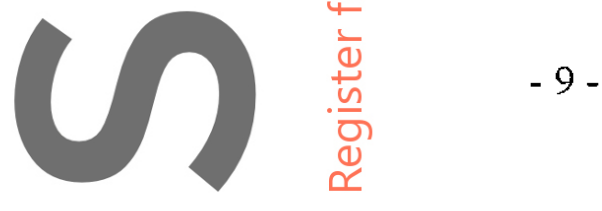




$$
r_{t}^{+}=\max \left\{r_{0}^{+}, \max _{s \in[0, t]}\left(\bar{\tau}_{s}^{+}\right)\right\}
$$

Introducing eqn (19b) into eqn (19a), during loading the evolution law for the tensile damage variable is expressible as

$$
\dot{d}^{+}=\frac{\partial G^{+}\left(r^{+}\right)}{\partial r^{+}} \dot{r}^{+}=\dot{G}^{+}\left(r^{+}\right) \geq 0
$$

For compression analogous kinematics will obviously result, that is:

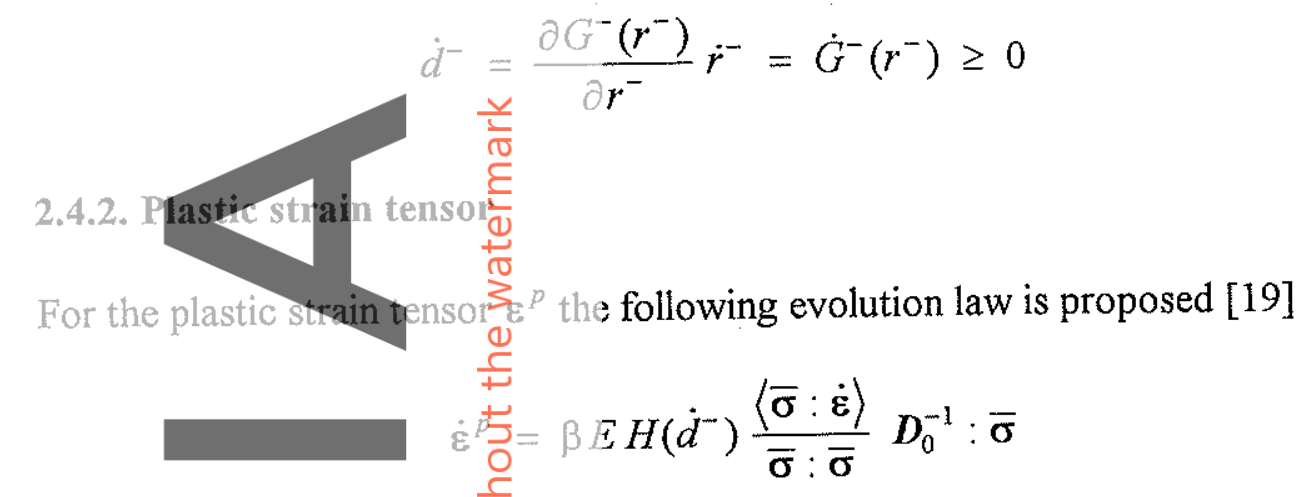

where, besides the Young's matulus $E$, a material parameter $\beta \geq 0$ is introduced, in order to control the rate intensity $\frac{\frac{c}{6}}{\frac{5}{d}}$ plastic deformation. $H\left(\dot{d}^{-}\right)$denotes the Heaviside step function, computed for the compressive damage rate. Macaulay brackets enable to set a non-negative value for the p pduct $\overline{\boldsymbol{\sigma}}: \dot{\boldsymbol{\varepsilon}}$, an essential thermodynamic requirement for ensuring a non-negative dissip $\underset{\frac{\pi}{\zeta}}{\stackrel{\pi}{\zeta}}$, as it will be demonstrated latter.

Through the definition $\frac{0}{0}$

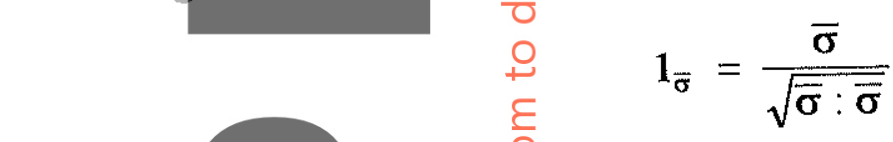

eqn (25) nay assume the compicted form

$$
\dot{\varepsilon}^{p} \cdot \stackrel{\cup}{\cup}_{\cup} \beta E H\left(\dot{d}^{-}\right)\left\langle 1_{\bar{\sigma}}: \dot{\boldsymbol{\varepsilon}}\right\rangle D_{0}^{-1}: \mathbf{1}_{\overline{\boldsymbol{\sigma}}}
$$

Remark 2.6. The basic ice equation (25) is that plastic strain evolution is assumed to have the 'direction' of the elastic strain tensor $D_{0}^{-1}: \bar{\sigma}$, which seems a reasonable assumptron (although obviously simplified), in the sense that plasticity is then essentially dreyen by the effective stress tensor $\bar{\sigma}$, an entity with physical background. Furthermore, 
non-linearity, damage and plasticity, avoids evolution of plastic strains during damage unloading or before the compressive damage threshold being attained (for instance, during the initial elastic branch or during a partial reloading), situations in which $H\left(\dot{d}^{-}\right)=0$.

\subsection{Clausius-Duhem Inequality}

During any loading process energy dissipation is always non-negative, which implies that entropy will tend to grow, hence leading to an irreversible process, according to the Second Principle of Thermodynamics. This condition from Thermodynamics of Irreversible Processes is commonly expressed by the Clausius-Duhem inequality $[18,23]$, whose reduced form is:

$$
\dot{\gamma}=-\dot{\psi}+\sigma: \dot{\varepsilon} \geq 0
$$

From eqns $(3,4)$ it is possible to express that

$$
\dot{\psi}=\frac{\partial \psi}{\partial \boldsymbol{\varepsilon}}: \dot{\boldsymbol{\varepsilon}}+\frac{\partial \psi}{\partial \boldsymbol{\varepsilon}^{p}}: \dot{\boldsymbol{\varepsilon}}^{p}+\frac{\partial \psi}{\partial d^{+}} \dot{d}^{+}+\frac{\partial \psi}{\partial d^{-}} \dot{d}^{-}
$$

result whose substitution into eqn (28), together with eqns (13), allows to obtain another expression for dissipation:

$$
\dot{\gamma}=\left(\boldsymbol{\sigma}-\frac{\partial \psi}{\partial \boldsymbol{\varepsilon}}\right): \dot{\boldsymbol{\varepsilon}}+\psi_{0}^{+} \dot{d}^{+}+\psi_{0}^{-} \dot{d}^{-}-\frac{\partial \psi}{\partial \boldsymbol{\varepsilon}^{p}}: \dot{\boldsymbol{\varepsilon}}^{p}
$$

\subsection{Constitutive Law}

Since $\varepsilon$ is a free variable, for the equation of dissipation to maintain its generality the expression within parenthesis in eqn (30) must cancel [18]. Hence

$$
\sigma=\frac{\partial \psi}{\partial \varepsilon}
$$

which constitutes one of the Coleman's relations, essential for the assessment of the constitutive law. 
Splitting the strain tensor $\varepsilon$ into $\varepsilon^{e}$ and $\varepsilon^{p}$, the elastic and plastic contributions, the effective stress tensor may be expressed as

$$
\bar{\sigma}\left(\varepsilon^{e}\right)=D_{0}:\left(\varepsilon-\varepsilon^{p}\right)=D_{0}: \varepsilon^{e}
$$

and consequently the elastic free energies defined by eqns (4) may assume the forms:

$$
\begin{aligned}
& \psi_{0}^{+}\left(\varepsilon^{e}\right)=\frac{1}{2} \bar{\sigma}^{+}: \varepsilon^{e} \\
& \psi_{0}^{-}\left(\varepsilon^{e}\right)=\frac{1}{2} \bar{\sigma}^{-}: \varepsilon^{e}
\end{aligned}
$$

Invoking the chain rule, and keeping in mind that $\varepsilon^{e}=\varepsilon-\varepsilon^{p}$, eqn (31) can be transformed into

$$
\boldsymbol{\sigma}=\frac{\partial \psi}{\partial \boldsymbol{\varepsilon}^{e}}: \frac{\partial \boldsymbol{\varepsilon}^{e}}{\partial \boldsymbol{\varepsilon}}=\frac{\partial \psi}{\partial \boldsymbol{\varepsilon}^{e}}
$$

and consequently, from eqn (3),

$$
\sigma=\left(1-d^{+}\right) \frac{\partial \psi_{0}^{+}}{\partial \varepsilon^{e}}+\left(1-d^{-}\right) \frac{\partial \psi_{0}^{-}}{\partial \varepsilon^{e}}
$$

Due to the linear dependency between $\bar{\sigma}$ and $\varepsilon^{e}$ (see eqn (32)), and taking into consideration the stress split expressed in eqns (2), it becomes clear that

$$
\bar{\sigma}^{+}\left(m \varepsilon^{e}\right)=m \bar{\sigma}^{+}\left(\varepsilon^{e}\right)
$$

for any arbitrary scalar $m$. This relation, and a similar one that can be verified for $\bar{\sigma}^{-}$, put into evidence that both $\bar{\sigma}^{+}$and $\bar{\sigma}^{-}$are first degree homogeneous functions of $\varepsilon^{e}$, and consequently, according to Euler's Theorem, it occurs that

$$
\bar{\sigma}^{+}\left(\varepsilon^{e}\right)=\frac{\partial \bar{\sigma}^{+}}{\partial \varepsilon^{e}}: \varepsilon^{e} \quad \bar{\sigma}^{-}\left(\varepsilon^{e}\right)=\frac{\partial \bar{\sigma}^{-}}{\partial \varepsilon^{e}}: \varepsilon^{e}
$$

In order to clarify the constitutive equation (35), eqn (33a) has to be derived with respect to $\varepsilon^{e}$ :

$$
\frac{\partial \psi_{0}^{+}}{\partial \varepsilon^{e}}=\frac{1}{2} \frac{\partial \bar{\sigma}^{+}}{\partial \varepsilon^{e}}: \varepsilon^{e}+\frac{1}{2} \bar{\sigma}^{+}
$$

Calling for eqn (37) it results:

$$
\frac{\partial \psi_{0}^{+}}{\partial \boldsymbol{\varepsilon}^{e}}=\bar{\sigma}^{+}
$$


For compression obviously it would be

$$
\frac{\partial \psi_{0}^{-}}{\partial \varepsilon^{e}}=\bar{\sigma}^{-}
$$

It is then possible to obtain a final form for the constitutive law (35), leading to a rather clear and intuitive expression for the $\sigma$ Cauchy stress tensor:

$$
\sigma=\left(1-d^{+}\right) \bar{\sigma}^{+}+\left(1-d^{-}\right) \bar{\sigma}^{-}
$$

\subsection{Dissipation}

In the expression of dissipation, eqn (30), in view of eqn (31) now reduced to

$$
\dot{\gamma}=\psi_{0}^{+} \dot{d}^{+}+\psi_{0}^{-} \dot{d}^{-}-\frac{\partial \psi}{\partial \varepsilon^{p}}: \dot{\varepsilon}^{p}
$$

first and second contributions are non-negative, since $\psi_{0}^{+}$and $\psi_{0}^{-}$are non-negative (see eqn (10)), and $\left(\dot{d}^{+}, \dot{d}^{-}\right) \geq 0$ due to the evolution laws adopted for the damage variables (eqns $(23,24)$ ). So, condition $\dot{\gamma} \geq 0$ will become demonstrated once proved the non-negativeness of the last contribution on dissipation in eqn (42). Demonstration will be performed keeping in mind that

$$
\frac{\partial \psi}{\partial \boldsymbol{\varepsilon}^{p}}=\frac{\partial \psi}{\partial \boldsymbol{\varepsilon}^{e}}: \frac{\partial \boldsymbol{\varepsilon}^{e}}{\partial \boldsymbol{\varepsilon}^{p}}=-\frac{\partial \psi}{\partial \boldsymbol{\varepsilon}^{e}}
$$

Moreover, in compacted form eqn (25) reduces to

$$
\dot{\boldsymbol{\varepsilon}}^{p}=b D_{0}^{-1}: \overline{\boldsymbol{\sigma}}
$$

with $b$ being the non-negative scalar

$$
b=\beta E H\left(\dot{d}^{-}\right) \frac{\langle\overline{\boldsymbol{\sigma}}: \dot{\varepsilon}\rangle}{\overline{\boldsymbol{\sigma}}: \overline{\boldsymbol{\sigma}}} \geq 0
$$

Taking into consideration eqns $(43,44)$, as well as eqns $(34,41)$, it can be concluded that

$$
-\frac{\partial \psi}{\partial \varepsilon^{p}}: \dot{\varepsilon}^{p}=b\left[\left(1-d^{+}\right) \bar{\sigma}^{+}: D_{0}^{-1}: \bar{\sigma}+\left(1-d^{-}\right) \bar{\sigma}^{-}: D_{0}^{-1}: \bar{\sigma}\right]
$$


Insight on the expression enclosed within the straight brackets, and comparison with eqns $(3,4)$, shows that this result may be expressed more concisely as

$$
-\frac{\partial \psi}{\partial \varepsilon^{p}}: \dot{\varepsilon}^{p}=2 b \psi
$$

therefore a non-negative quantity since $(\psi, b) \geq 0$, which ensures that the Clausius-Duhem dissipation inequality applies:

$$
\dot{\gamma}=\psi_{0}^{+} \dot{d}^{+}+\psi_{0}^{-} \dot{d}^{-}+2 b \psi \geq 0
$$

\subsection{Numerical Computation of the Internal Variables}

Owing to the strain-driven formalism of the proposed constitutive model, and to the fact of $\varepsilon$ being fully determined at the beginning of each step of a displacement-based finite element analysis, the updating of the internal variables becomes possible via efficient and almost direct algorithms, as it will be illustrated next.

\subsubsection{Plastic strain tensor}

Performing a temporal derivative of eqn (1), where eqn (25) is also taken into account, it results:

$$
\dot{\bar{\sigma}}=D_{0}: \dot{\varepsilon}-\beta E H\left(\dot{d}^{-}\right)\langle\bar{\sigma}: \dot{\varepsilon}\rangle \frac{\bar{\sigma}}{\bar{\sigma}: \bar{\sigma}}
$$

A temporal discretization based on a 'backward-Euler' scheme may then be adopted, where $(\cdot)_{n}$ and ()$_{n+1}$ denote entities referred to consecutive time steps, and $\Delta \varepsilon$ refers to the associated increment in $\varepsilon$ :

$$
\bar{\sigma}_{n+1}=\bar{\sigma}_{n}+D_{0}: \Delta \varepsilon-\beta E H\left(\dot{d}_{n+1}^{-}\right)\left\langle\bar{\sigma}_{n+1}: \Delta \varepsilon\right\rangle \frac{\bar{\sigma}_{n+1}}{\bar{\sigma}_{n+1}: \bar{\sigma}_{n+1}}
$$

Defining

$$
\begin{aligned}
& \overline{\boldsymbol{\sigma}}_{n+1}^{\text {trial }}=\overline{\boldsymbol{\sigma}}_{n}+D_{0}: \Delta \varepsilon \\
& \|\overline{\boldsymbol{\sigma}}\|=\sqrt{\bar{\sigma}: \bar{\sigma}}
\end{aligned}
$$

eqn (50) may assume the form 


$$
\left\{\frac{\left\|\bar{\sigma}_{n+1}\right\|^{2}+\beta E H\left(\dot{d}_{n+1}^{-}\right)\left\langle\bar{\sigma}_{n+1}: \Delta \varepsilon\right\rangle}{\left\|\bar{\sigma}_{n+1}\right\|}\right\} \frac{\bar{\sigma}_{n+1}}{\left\|\bar{\sigma}_{n+1}\right\|}=\bar{\sigma}_{n+1}^{\text {trial }}
$$

Introducing the normalised tensor $1_{\bar{\sigma}}$ defined in eqn (26), as well as the auxiliary variable

$$
\Phi=\left\|\bar{\sigma}_{n+1}\right\|+\beta E H\left(\dot{d}_{n+1}^{-}\right)\left\langle\mathbf{1}_{\bar{\sigma}_{n+1}}: \Delta \varepsilon\right\rangle
$$

eqn (53) is equivalent to

$$
\Phi 1_{\bar{\sigma}_{n+1}}=\bar{\sigma}_{n+1}^{\text {trial }}
$$

and consequently $\Phi=\left\|\bar{\sigma}_{n+1}^{\text {trial }}\right\|$. This result is useful to rewrite eqn (55) as

$$
\Phi 1_{\bar{\sigma}_{n+1}}=\left\|\overline{\boldsymbol{\sigma}}_{n+1}^{\text {trial }}\right\| 1_{\overline{\boldsymbol{\sigma}}_{n+1}^{\text {trial }}}=\Phi 1_{\overline{\boldsymbol{\sigma}}_{\text {trial }}^{\text {triat }}}
$$

which leads to the conclusion that $1_{\vec{\sigma}_{n+1}}=1_{\bar{\sigma}_{n+1}^{\text {rial }}}$, and so eqn (54) may be transformed into

$$
\left\|\overline{\boldsymbol{\sigma}}_{n+1}\right\|=\left\|\overline{\boldsymbol{\sigma}}_{n+1}^{\text {trial } \|}\right\|-\beta E H\left(\dot{d}_{n+1}^{-}\right)\left\langle 1_{\overline{\boldsymbol{\sigma}}_{n+1}^{\text {rrial }}}: \Delta \varepsilon\right\rangle
$$

Owing to these results, the effective stress tensor can be updated according to

$$
\overline{\boldsymbol{\sigma}}_{n+1}=\left\|\overline{\boldsymbol{\sigma}}_{n+1}\right\| 1_{\overline{\boldsymbol{\sigma}}_{n+1}^{\text {trial }}}=\lambda \overline{\boldsymbol{\sigma}}_{n+1}^{\text {trial }}
$$

where

$$
\lambda=1-\frac{\beta}{\left\|\overline{\boldsymbol{\sigma}}_{n+1}^{\text {trial }}\right\|} E H\left(\dot{d}_{n+1}^{-}\right)\left\langle\mathbf{1}_{\bar{\sigma}_{n+1}^{\text {tral }}}: \Delta \mathbf{\varepsilon}\right\rangle
$$

Note that $\Delta \varepsilon, \bar{\sigma}_{n+1}^{\text {trial }}$ and $\mathbf{1}_{\overline{\boldsymbol{\sigma}}_{n+1}^{\text {trial }}}$ are known in terms of the strains in step $n+1$. From eqn (58) it becomes clear that a 'radial return' procedure can be set up for the updating of $\bar{\sigma}_{n+1}$ : tensor $\bar{\sigma}_{n+1}^{\text {trial }}$ can be looked as a prediction, through which the effective stress tensor can be obtained once evaluated the scale factor $\lambda$, the unique entity to be determined. Due to the $0 / 1$ discontinuity introduced in eqn (59) by the Heaviside function, a double iterative algorithm may be required, which is illustrated in Box 1.

It must be remarked that from eqn (57) the inequality $\left\|\bar{\sigma}_{n+1}\right\| \leq\left\|\bar{\sigma}_{n+1}^{\text {trial }}\right\|$ applies. As according to eqn (58) tensors $\bar{\sigma}_{n+1}$ and $\bar{\sigma}_{n+1}^{\text {trial }}$ are proportional, in view of the stress split 
expressed in eqns (2) their compressive components are also affected by the same scale factor, that is, $\left(\bar{\sigma}_{n+1}\right)^{-}=\lambda\left(\bar{\sigma}_{n+1}^{t r i a l}\right)^{-}$, with $0 \leq \lambda \leq 1$. Consequently, denoting by $\vec{\tau}^{-}\left(\left(\bar{\sigma}_{n+1}^{\text {trial }}\right)^{-}\right)$the equivalent stress associated to the negative component $\left(\bar{\sigma}_{n+1}^{\text {trial }}\right)^{-}$, it becomes clear that $\bar{\tau}_{n+1}^{-} \leq \bar{\tau}^{-}\left(\left(\bar{\sigma}_{n+1}^{t r i a l}\right)^{-}\right)$. This means that if $\bar{\tau}^{-}\left(\left(\bar{\sigma}_{n+1}^{\text {trial }}\right)^{-}\right)<r_{n}^{-}$it occurs that $\bar{\tau}_{n+1}^{-}<r_{n}^{-}$, that is, $\dot{d}_{n+1}^{-}=0$, and consequently no plastic evolution takes place; in this situation $\lambda=1$ (see eqn (59), with $H\left(\dot{d}_{n+1}^{-}\right)=0$ ), which explains the EXIT appearing in step (iii) of Box 1 .

Box 1. Algorithm for the determination of $\bar{\sigma}_{n+1}$.

(i) Compute $\bar{\sigma}_{n+1}^{t r i a l}=\bar{\sigma}_{n}+D_{0}: \Delta \varepsilon$

(ii) Is $\beta=0$ ?

YES: No plasticity. Set $\overline{\boldsymbol{\sigma}}_{n+1}=\overline{\boldsymbol{\sigma}}_{n+1}^{\text {trial }}$. EXIT.

NO : GO TO (iii)

(iii) Split $\bar{\sigma}_{n+1}^{\text {trial }}$ into $\left(\bar{\sigma}_{n+1}^{\text {trial }}\right)^{+}$and $\left(\bar{\sigma}_{n+1}^{\text {trial }}\right)^{-}$. Evaluate $\bar{\tau}^{-}\left(\left(\bar{\sigma}_{n+1}^{\text {trial }}\right)^{-}\right)$with eqn (14b).

Is $\bar{\tau}^{-}\left(\left(\bar{\sigma}_{n+1}^{\text {trial }}\right)^{-}\right)<r_{n}^{-}$?

YES: No evolution for $d^{-}$and $\varepsilon^{p}$. Set $\bar{\sigma}_{n+1}=\bar{\sigma}_{n+1}^{\text {trial }}$. EXIT.

NO : GO TO $(i v)$.

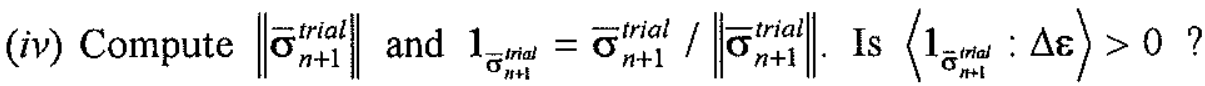

YES: Plastic evolution is possible. Admitting $H\left(\dot{d}_{n+1}^{-}\right)=1$, compute $\lambda$ with eqn (59) and $\hat{\bar{\sigma}}=\lambda \bar{\sigma}_{n+1}^{\text {trial }}$. GO TO $(v)$.

NO : No plastic evolution. Set $\bar{\sigma}_{n+1}=\bar{\sigma}_{n+1}^{\text {trial }}$. EXIT

(v) Split $\hat{\bar{\sigma}}$ into $\hat{\bar{\sigma}}^{+}$and $\hat{\bar{\sigma}}^{-}$. Evaluate $\bar{\tau}^{-}\left(\hat{\bar{\sigma}}^{-}\right)$with eqn (14b). Is $\bar{\tau}^{-}\left(\hat{\bar{\sigma}}^{-}\right)<r_{n}^{-}$?

YES: No evolution for $d^{-}$and $\varepsilon^{p}$. Set $\bar{\sigma}_{n+1}=\bar{\sigma}_{n+1}^{\text {trial }}$. EXIT.

NO : Evolution of $d^{-}$and $\varepsilon^{p}$ exists. Set $\bar{\sigma}_{n+1}=\hat{\bar{\sigma}}$. EXIT.

\subsubsection{Damage variables}

Performing a trivial integration, the rate eqns $(23,24)$ lead to the following expressions 
with

$$
d^{+}=G^{+}\left(r^{+}\right) \quad d^{-}=G^{-}\left(r^{-}\right)
$$

$$
\begin{array}{rlrl}
0 \leq G^{+}\left(r^{+}\right) & \leq 1 & 0 \leq G^{-}\left(r^{-}\right) & \leq 1 \\
\dot{G}^{+}\left(r^{+}\right) & \geq 0 & \dot{G}^{-}\left(r^{-}\right) & \geq 0 \\
G^{+}\left(r_{0}^{+}\right) & =0 & G^{-}\left(r_{0}^{-}\right) & =0
\end{array}
$$

It can be inferred that once the strain tensor is known the damage variables can be easily computed, since $r^{+}$and $r^{-}$depend on $\varepsilon$. Note that condition (11) is introduced in eqns (60b). Eqns (60c) state the positive evolutions for the damage variables expressed in eqns $(23,24)$, ensuring $G^{+}(\cdot)$ and $G^{-}(\cdot)$ to be monotonically increasing functions. Finally, eqns (60d) introduce the initial conditions of null damages.

The main concerns for the next issues are the particular forms to attribute to the scalar functions $G^{+}$and $G^{-}$. In the present work the following evolution rule will be adopted to reproduce the softening branch of a test performed under $1 D$ tension [24]

$$
d^{+}=G^{+}\left(r^{+}\right)=1-\frac{r_{0}^{+}}{r^{+}} e^{A^{+}\left(1-r^{+} / r_{0}^{+}\right)} \quad \text {, if } \quad r^{+} \geq r_{0}^{+}
$$

involving $A^{+}$as unique parameter. This formula leads to a curve asymptotic to the strain axis, and consequently parameter $A^{+}$must be fixed taking into consideration requisites of mesh-objectivity. If finite element analyses are to be performed, in the context of local models a geometrical 'characteristic length' $l_{c h}$ is commonly introduced [25], depending on the size (volume or area) of the elements adopted for the spatial discretization. By equating $G_{f} / l_{c h}$ to the finite area retained under the stress-strain curve, where $G_{f}$ denotes the tensile fracture energy (assumed to be a material property), parameter $A^{+}$ can be set according to [24]:

$$
A^{+}=\left(\frac{G_{f} E}{l_{c h}\left(f_{0}^{+}\right)^{2}}-\frac{1}{2}\right)^{-1} \geq 0
$$

Under compression the evolution of damage will be simulated through the expression underneath, which is inspired on a formula presented in [15]:

$$
d^{-}=G^{-}\left(r^{-}\right)=1-\frac{r_{0}^{-}}{r^{-}}\left(1-A^{-}\right)-A^{-} e^{B^{-}\left(1-r^{-} / r_{0}^{-}\right)} \quad \text { if } \quad r^{-} \geq r_{0}^{-}
$$


Parameters $A^{-}$and $B^{-}$may be defined by imposing the $\sigma-\varepsilon$ ID numerical curve to convey two selected points on a curve extracted from a $1 \mathrm{D}$ compressive test ${ }^{\dagger}$.

\subsection{Numerical Integration of the Constitutive Law}

The implementation of the plastic-damage constitutive model is illustrated by Box 2 , which shows all the operations needed for evaluating the Cauchy stress tensor. A remark is made for the elegance and readability of the overall algorithm, as well as for the simplicity of the involved step-by-step operations, a consequence of the adopted strain-driven strategy, which provides an easy code implementation and contributes to improve the computational efficiency.

Box 2. Algorithm for the plastic-damage model.

Step $n=0$ :

(i) Set $r_{n}^{+}=r_{0}^{+}, r_{n}^{-}=r_{0}^{-}, d_{n}^{+}=0$ and $d_{n}^{-}=0$.

\section{Step $n+1$ :}

(ii) Evaluate $\varepsilon_{n+1}$. Compute $\bar{\sigma}_{n+1}$ according to Box 1 .

(iii) Split $\bar{\sigma}_{n+1}$ into $\bar{\sigma}_{n+1}^{+}$and $\bar{\sigma}_{n+1}^{-}$.

(iv) Compute $\bar{\tau}_{n+1}^{+}$and $\bar{\tau}_{n+1}^{-}$according to eqns (14).

(v) If $\bar{\tau}_{n+1}^{+}>r_{n}^{+}$or $\bar{\tau}_{n+1}^{-}>r_{n}^{-}$update damage thresholds: $r_{n+1}^{+}=\max \left\{r_{n}^{+}, \bar{\tau}_{n+1}^{+}\right\}$ or $r_{n+1}^{-}=\max \left\{r_{n}^{-}, \bar{\tau}_{n+1}^{-}\right\}$.

Update damage variables $d_{n+1}^{+}=G^{+}\left(r_{n+1}^{+}\right)$and $d_{n+1}^{-}=G^{-}\left(r_{n+1}^{-}\right)$, according to eqns (61) and (63).

(vi) Compute the Cauchy stress tensor

$$
\sigma_{n+1}=\left(1-d_{n+1}^{+}\right) \bar{\sigma}_{n+1}^{+}+\left(1-d_{n+1}^{-}\right) \bar{\sigma}_{n+1}^{-} \quad \text { EXIT. }
$$

$\dagger$ If softening is also expected in compression, parameters $A^{-}$and $B^{-}$may also be attributed so as to satisfy requirements of mesh-objectivity. A mesh-dependent characteristic length and a fracture energy for compression could also be invoked, similarly as already described for tension. 
Fig. 3 depicts the typical performance of the constitutive model during a 1D tension-compression cyclic test, performed over an idealised concrete specimen. A complex loading scheme was imposed, comprising an incursion into tensile regimen up to the initial elastic threshold (path $\mathrm{O}-\mathrm{A}$ ), and leading to damage thereafter, along path A-B. Loading is then reversed, producing a return to point $\mathrm{O}$ and a subsequent incursion into compression up to threshold $\mathrm{C}$. From there until point $\mathrm{D}$ progressive damage and plastic deformation is observed; at point $\mathrm{D}$ a new load reversal is enforced, originating the broken line D-E-F-G. Between points $F$ and $G$ further tensile damage occurs.

It becomes visible the ability of the plastic-damage model to reproduce the softening behaviour under tension, as well as for capturing the hardening and softening which occurs in concrete under compression. An incursion into the tensile regimen without previous induced compressive damage does not allow plastic strains to take place, as evidenced during the first tensile unloading (straight line B-O). Note also the stiffness recovery that takes place during paths B-O-C, D-E-F or G-E-D: this 'unilateral effect' corresponds to a peculiar feature of concrete behaviour, fully captured by the proposed model, owing to its 'memory' proficiency. Moreover, the constitutive model has also the capability of maintaining the plastic deformations induced during previous compressive damaging, as demonstrated by the horizontal shift E-O experienced by curve E-F-G, corresponding to an irreversible strain.

\subsection{Applications}

\subsubsection{D cyclic test in compression}

The plastic-damage model ability for reproducing the typical concrete behaviour during a 1D cyclic compressive test can be checked in Fig. 4, where experimental results taken from Ref. [26] (according to [27]) are plotted against the numerical predictions. The properties considered for the concrete, with a $32 \mathrm{MPa}$ compressive peak strength, were: $E=26 \mathrm{GPa}, f_{0}^{-}=15 \mathrm{MPa}, \beta=0.590$. As it can be observed, predictions from the 
numerical model agree fairly well with the experimental results, namely in what concerns to: $(i)$ the overall non-linear behaviour evidenced by the calculated envelope curve, either in the hardening or in the softening regimens, which is rather close to the test one; (ii) the residual plastic strain upon unloading, which is continuously increasing as further straining takes place, in accordance with the observed experimental behaviour; (iii) the progressive degradation of the secant modulus, expressing that continuous damage is occurring, which reproduces rather well the 'average' lines from the test unloading-reloading loops. Therefore, the evolution laws adopted for damage and plasticity seem to be physically realistic and adequate for modelling the compressive behaviour of a concrete specimen under a $1 \mathrm{D}$ cyclic test.

\subsubsection{D test in compression}

Fig. 5 refers to a set of experimental tests reported in Ref. [22], performed with concrete specimens under $2 \mathrm{D}$ compression $\left(\sigma_{1}=0\right)$, according to the following load conditions: $\left(\right.$ i) $\sigma_{3} / \sigma_{2}=-1 / 0 ;$ (ii) $\sigma_{3} / \sigma_{2}=-1 /-1$ and (iii) $\sigma_{3} / \sigma_{2}=-1 /-0.52$. The properties adopted for the plastic-damage model were: $E=31 \mathrm{GPa}, \quad v=0.2$, $f_{0}^{-}=10 \mathrm{MPa}$ and $\beta=0.318$. As it can be noticed, model curves exhibit an acceptable agreement with the test ones, capturing satisfactorily the overall experimental behaviour, specially taking into consideration the purposes intended within the context of large scale computations, and the simplifications which had been introduced for the improvement of the computational efficiency. An important attribute of the present constitutive model, clearly perceptible in Fig. 5, is the ability to predict the concrete strength enhancement under 2D compression. As it can be inferred from the state-of-art included in Ref. [15], this feature, with an evident relevance for concrete behaviour, was not captured by older versions of similar damage models, therefore constituting a significant improvement from the present model. 


\subsubsection{D test in compression}

As a third benchmark, Fig. 6 compares numerical predictions with the experimental results reported in [28] for concrete under $3 \mathrm{D}$ compression. Tests were driven with an increasing normal stress along the specimen vertical axis, and three different sets of confining stresses along the horizontal directions: (i) $\sigma_{1}=\sigma_{2}=0.0 \mathrm{MPa}$; (ii) $\sigma_{1}=\sigma_{2}=-6.895 \mathrm{MPa}$ and $($ iii $) \sigma_{1}=\sigma_{2}=-13.790 \mathrm{MPa}$. The following properties were adopted for concrete: $E=41.37 \mathrm{GPa}, u=0.2, f_{0}^{-}=10 \mathrm{MPa}$ and $\beta=0$. According to Fig. 6, the overall response curves, as well as the strength and ductility enhancements produced by the 3D confinement, are satisfactorily captured by the damage model, whose deviations from the experimental results may be considered acceptable, within the scope of the intended purposes of the present constitutive model.

\subsubsection{Representative structural applications}

As damage variables are intuitively associated with physical deterioration, the output from the model, namely through the superposition to the concrete body of the predicted damage patterns, provides an interesting tool for an overall interpretation of the structural behaviour, including the identification of failure mechanisms.

An illustration of this, for the arch type Foz Côa dam designed to be built in the north of Portugal ( $136 \mathrm{~m}$ high, $430 \mathrm{~m}$ crest length), may be observed in Fig. 7. Here the state of the dam after the occurrence of a $1 \mathrm{~g}$ peak acceleration earthquake is reproduced in terms of the final distribution for the $d^{-}$damage variable, relevant for the assessment of the concrete performance under compression, which is vital for the safety of this kind of structures (further details about the whole seismic analysis can be found in Ref. [29]). It becomes clear that compressive damage is only visible for a restricted concrete domain around the centre of the crest arch; close to the foundations (a relevant part for structural stability) concrete remains practically unaffected. Concrete volumes exhibiting damage $d^{-}$are therefore very limited, and so no collapse would be eminent for Foz Côa dam due to the selected seism. It is remarked the utility of this kind of information, linked to the distribution of damages, through which a physically comprehensible justification for the 
well-known ability of arch dams to withstand intense earthquakes is easily and naturally obtained.

Despite the plastic-damage model being mainly intended for the analysis of unreinforced massive concrete, through the next example it will be demonstrated its applicability for the analysis of a reinforced concrete beam, tested and reported in [30] under the designation of A3. Following this reference, the simply supported beam A3 was submitted to an increasing point load $F$ at mid-span, as depicted in Fig. 8a, which also details the most relevant dimensions and steel reinforcements, as well as the $2 \mathrm{D}$ and 1D discretizations adopted for the concrete and the steel bars, respectively (for additional informations concerning the overall analysis and the properties selected for the two materials, the reader is addressed to Ref. [29]). According to Fig. 8b, which describes the evolution of load $F$ with the mid-span vertical displacement $\Delta$, an acceptable agreement is observed between the numerical predictions and the structural behaviour experimentally registered for the beam; the collapse load obtained numerically was $9 \%$ lower than the test one. Fig. $8 \mathrm{c}$ documents the distribution for the tensile damage variable $d^{+}$obtained at the ultimate load, and illustrates the ability from the numerical model to predict the concrete fractured region, in accordance with the experimental evidence.

\section{EXTENSION TO STRAIN-RATE DEPENDENCY}

\subsection{Overview}

As widely recognised, concrete exhibits strain-rate sensitivity during dynamic tests [31-34], whose visible effects (when compared to quasi-static tests) are substantial gains in the peak strengths, as well as decreases of the stress-strain non-linearities. This singular behaviour is rather important under impulsive loading, like in impacts or explosions [35], but the intuition that the phenomenon is already important under the earthquake loading is widely generalized, as straining rates as high as 
$10^{-6} / \mathrm{s}<\dot{\varepsilon}<10^{-1} / \mathrm{s}$ may then be expected, leading to strength enhancements up to $80 \%$ in tension and $25 \%$ in compression.

The physical relevance of this viscous phenomenon has been identified long time ago, but its intrinsic complexity precluded it from common usage, the customary practice being to account for rate-sensitivity through the inclusion of drastic simplifications on the numerical models, like the a priori assumption, for dynamic purposes, of artificially increased values for strengths and elastic modulus. First attempts to deal with rate-dependency with theoretical consistency came from Viscoplasticity, like in Ref. [36]. More recently important experimental and theoretical contributions have been presented in [31-34], namely through the proposal of a constitutive model based on Continuum Damage Mechanics (CDM), with a vectorial representation for the internal damage.

According to [33], concrete rate sensitivity is mainly due to the fact of the growth of internal microcracking being retarded at high strain-rates, reducing the macroscopic non-linear behaviour. For the purposes intended within the context of the present work, comprising the analysis of large scale structures, an algorithm should evolve from the previous inviscid version, keeping its closed-form nature as much as possible, in order to maintain the high computational efficiency indispensable for the calculations to be feasible. Owing to the coupling between rate-sensitivity and damage, CDM provides an appropriate environment for dealing with this viscous behaviour, herein after accounted for by introducing a viscous regularization into the rate-independent evolution laws presented in Section 2.4.1 for the damage thresholds.

\subsection{Viscous regularization}

Adopting a procedure resembling the classic regularization suggested by Perzina in the framework of Viscoplasticity, the evolution of the damage threshold $r^{+}$described through eqn (19b) will be replaced by (see also $[19,37]$ )

$$
\dot{r}^{+}=\mu^{+} \phi^{+}\left(\vec{\tau}^{+}, r^{+}\right) \geq 0
$$




$$
\phi^{+}\left(\bar{\tau}^{+}, r^{+}\right)=r_{0}^{+}\left(\frac{\left\langle\bar{\tau}^{+}-r^{+}\right\rangle}{r^{+}}\right)^{a^{+}}
$$

where $\phi^{+}$may be termed a viscous damage threshold flow function. $\mu^{+}$denotes a fluidity parameter, while $a^{+}$is a positive exponent, both assumed to be material properties, which can be evaluated on the basis of $1 \mathrm{D}$ tensile tests. As for the tensile damage variable itself, the previous evolution law expressed in eqn (23) is kept unchanged, and consequently $d^{+}$may be updated with the same efficiency as for the inviscid model, through eqn (61) which fixes $d^{+}=G^{+}\left(r^{+}\right)$.

If compression is to be considered, a set of equations similar to (64) can be postulated, with distinct material parameters $\left(\mu^{-}, a^{-}\right)$and flow function $\phi^{-}$being then attributed, permitting to attend for the different rate-sensitivity exhibited by concrete under compressive loading, in comparison to what occurs under tension. Explicit computation of variable $d^{-}$through the inviscid function $G^{-}\left(r^{-}\right)$, eqn (63), maintains its validity.

Remark 3.1. For the sake of simplicity, throughout the present extension to strain-rate dependency no plastic evolution is accounted for ${ }^{\dagger}$. Following Section 2.7, dissipation may be expressed through eqn (42), with the last term on the right-hand side vanishing for the present strictly viscous-damage constitutive model. The only request from the Clausius-Duhem inequality is therefore $\left(\dot{d}^{+}, \dot{d}^{-}\right) \geq 0$, which is trivially ensured by the kinematics for the damage variables expressed in equations like (61) and (63). As for the constitutive law itself, eqn (41) remains valid for the present rate-dependent model.

Remark 3.2. According to eqn (64a), through the setting of a null value to a fluidity parameter the evolution of the associated damage threshold is prevented, thus enforcing

† The constitutive model is therefore strictly viscous-damage, although no important difficulties would arise to account for viscous-plasticity if a Perzina-type regularization was also introduced into the kinematics for the plastic strain tensor, as usual practice in Viscoplasticity. 
a linear-elastic response to be obtained. On the other hand, as $\mu^{+}$and $\mu^{-}$approach to infinity the rate-independent damage evolution laws are recovered, once

$$
\mu \rightarrow \infty \Rightarrow(\phi=\dot{r} / \mu) \rightarrow 0 \Rightarrow\left\{\begin{array}{l}
\bar{\tau} \rightarrow r \\
\dot{\bar{\tau}} \rightarrow \dot{r}
\end{array}\right.
$$

These reasonings demonstrate that the proposed viscid formulation provides a general framework for the threshold and damage evolutions, including both the linear-elasticity and the previous damage model.

Remark 3.3. As for the rate-independent model, mesh-objectivity requisites under softening responses must also be fulfilled throughout the present viscous-type extension. For a proper localization to be obtained, and in order to ensure mesh independent time responses, the fluidity parameter must also be defined as function of the characteristic length [38]. For the present viscous-damage model this effect can be attended if $\mu^{+}$in eqn (64a) is defined in accordance with (see [37], for details)

$$
\mu^{+}=\bar{\mu}^{+}\left(\frac{1}{l_{c h}}-\frac{\left(f_{0}^{+}\right)^{2}}{2 E G_{f}}\right) \geq 0
$$

where $\bar{\mu}^{+}$is assumed to be a material property. A similar strategy could be adopted for $\mu^{-}$, if softening might also be expected in compression.

\subsection{Numerical Implementation}

Integration of the Cauchy stress tensor in time requires an appropriate algorithm to update the damage variables and thresholds, defined in accordance with the kinematic equations, preferably with the most closed-form structure as possible.

According to eqns (64), the real numerical problem to be solved is the determination of thresholds $r^{+}$and $r^{-}$. Setting a marching scheme such that $(\cdot)_{n+1}$ denotes entities respecting to the actual time, $(\cdot)_{n}$ refers to entities already determined in a previous instant, and $\Delta t$ designates the time step, equations like (64a) may be integrated as follows, using a generalized mid-point rule 


$$
r_{n+1}=r_{n}+\Delta t \mu \phi\left(\bar{\tau}_{\alpha}, r_{\alpha}\right)
$$

with

$$
\begin{array}{ll}
\bar{\tau}_{\alpha}=(1-\alpha) \bar{\tau}_{n}+\alpha \bar{\tau}_{n+1} & \\
r_{\alpha}=(1-\alpha) r_{n}+\alpha r_{n+1} & (\alpha \in[0.5,1.0]) \dagger
\end{array}
$$

Rearranging eqn (66) taking into consideration eqns (64b) and (67), it results

$$
f\left(r_{n+1}\right)=-r_{n+1}+r_{n}+\Delta t \mu r_{0}\left(\left\langle\bar{\tau}_{\alpha}-r_{\alpha}\right\rangle / r_{\alpha}\right)^{a}=0
$$

For $a \neq 1$ this equation is non-linear, and consequently an explicit solver for $r_{n+1}$ only exists when exponent $a$ is an integer not greater than 4. If greater (or real) exponents are to be used, an elemental iterative Newton-Raphson scheme may be adopted to extract $r_{n+1}$ from eqn (68), and so a recursive formula like

$$
r_{n+1}^{i+1}=r_{n+1}^{i}-\frac{f\left(r_{n+1}^{i}\right)}{f^{\prime}\left(r_{n+1}^{i}\right)}
$$

allows to obtain an $(i+1)$-th improved approximation for $r$, taking into consideration information from a previous $(i)$-th iteration. $f^{\prime}$ designates the first derivative of $f$, that is

$$
f^{\prime}\left(r_{n+1}\right)=-1-H\left(\bar{\tau}_{\alpha}-r_{\alpha}\right) a \alpha \Delta t \mu r_{0} \frac{\bar{\tau}_{\alpha}}{\left(r_{\alpha}\right)^{2}}\left(\left(\bar{\tau}_{\alpha}-r_{\alpha}\right) / r_{\alpha}\right)^{a-1}
$$

Note the presence of the Heaviside function in this equation, expressing that threshold $r_{n+1}$ will be updated only if $\bar{\tau}_{\alpha}>r_{\alpha}$, condition which comes from eqn (64b). As determination of $r_{\alpha}$ requires the computation of $r_{n+1}$, which is unknown before completion of the iterative process itself, the sequence of approximations expressed through eqn (69) can only be obtained on the basis of some conjecture on $H\left(\bar{\tau}_{\alpha}-r_{\alpha}\right)$, which is checked (and eventually corrected) at the end of the time step. As computation of $\bar{\tau}_{\alpha}$ is direct, comparison of this equivalent stress with $r_{n}$ provides relevant information for this conjecture: $(i)$ if $\bar{\tau}_{\alpha}<r_{n}$ it also occurs that $\bar{\tau}_{\alpha}<r_{\alpha}$, and so $H\left(\bar{\tau}_{\alpha}-r_{\alpha}\right)=0$, the

According to elementary numerical analysis, an unconditionally stable algorithm is obtained for $\alpha \geq 0.5$. The first order accurate backward-Euler difference scheme is reproduced for $\alpha=1.0$, whilst the second-order accurate trapezoidal rule is obtained for $\alpha=0.5$. 
iterative scheme being then dispensable (that is, $r_{n+1}=r_{n}$ ); (ii) if $\bar{\tau}_{\alpha}>r_{n}$ it is supposed that $H\left(\bar{\tau}_{\alpha}-r_{\alpha}\right)=1$.

For the overall comprehension on how the integration of the viscous-damage model can be performed, Box 3 describes the basic operations to be accounted for on a computational code.

Box 3. Algorithm for the viscous-damage model.

\section{Step $n=0$ :}

(i) Set $r_{n}^{+}=r_{0}^{+}, r_{n}^{-}=r_{0}^{-}, d_{n}^{+}=0$ and $d_{n}^{-}=0$.

\section{Step $n+1$ :}

(ii) Evaluate $\varepsilon_{n+1}$. Compute $\bar{\sigma}_{n+1}=D_{0}: \varepsilon_{n+1}$.

(iii) Split $\bar{\sigma}_{n+1}$ into $\bar{\sigma}_{n+1}^{+}$and $\bar{\sigma}_{n+1}^{--}$.

(iv) Compute $\bar{\tau}_{n+1}^{+}$and $\bar{\tau}_{n+1}^{-}$according to eqns (14).

(v) Compute $\bar{\tau}_{\alpha}^{+}$and $\bar{\tau}_{\alpha}^{-}$according to eqn (67a). Is $\bar{\tau}_{\alpha}^{+}<r_{n}^{+}$(or $\bar{\tau}_{\alpha}^{-}<r_{n}^{-}$)?

YES: No threshold evolution. Set $r_{n+1}^{+}=r_{n}^{+}$(or $r_{n+1}^{-}=r_{n}^{-}$). GO TO (vi).

NO : Compute $r_{n+1}^{+}$and $r_{n+1}^{-}$according to eqns (68-70), admitting $H(\cdot)=1$.

Evaluate $r_{\alpha}^{+}$and $r_{\alpha}^{-}$according to eqn (67b). Is $\bar{\tau}_{\alpha}^{+}<r_{\alpha}^{+}$(or $\bar{\tau}_{\alpha}^{-}<r_{\alpha}^{-}$)? YES: Reset $r_{n+1}^{+}=r_{n}^{+}$(or $r_{n+1}^{-}=r_{n}^{-}$). GO TO (vi).

NO : GO TO $(v i)$.

(vi) Update damage variables $d_{n+1}^{+}=G^{+}\left(r_{n+1}^{+}\right)$and $d_{n+1}^{-}=G^{-}\left(r_{n+1}^{-}\right)$.

(vii) Compute the Cauchy stress tensor

$$
\sigma_{n+1}=\left(1-d_{n+1}^{+}\right) \bar{\sigma}_{n+1}^{+}+\left(1-d_{n+1}^{-}\right) \bar{\sigma}_{n+1}^{-} \quad \text { EXIT. }
$$

\subsection{Applications}

The performance of the rate-dependent model under straining rates between $10^{-6} / \mathrm{s}$ and $1 / \mathrm{s}$ is illustrated in Fig. 9, for a concrete specimen loaded in 1D tension. As it can be 
qualitatively observed, peak strengths grow continuously as straining rates are increased, becoming clearly distinguishable from the quasi-static one (approximately coincident with the curve denoted by $\dot{\varepsilon}=10^{-6} / \mathrm{s}$ ).

The viscous-damage model ability to reproduce the $1 \mathrm{D}$ experimental behaviour of concrete specimens, loaded either in tension or in compression, may be checked via Fig. 10. Coefficient $\rho$ appearing in this figure designates the ratio between the dynamic and the quasi-static peak strengths, and is plotted against the straining rate $\dot{\varepsilon}$, as reported in [33]. For the numerical simulation material properties were selected as follows: $E=30 \mathrm{GPa}, G_{f}=250 \mathrm{Jm}^{-2}, f_{0}^{+}=2 \mathrm{MPa}, f_{0}^{-}=12 \mathrm{MPa}, A^{+}=0.677, A^{-}=1.000$, $B^{-}=0.890, \bar{\mu}^{+}=870 \mathrm{~s}^{-1}, \bar{\mu}^{-}=40000 \mathrm{~s}^{-1}, a^{+}=a^{-}=5$. As it becomes clear from the analysis of Fig. 10, predictions from the viscid model are in fairly good agreement with the experimental results, both for tensile or compressive loading conditions.

\section{CLOSURE}

This article was devoted to the presentation of a new constitutive model, mainly intended for the analysis of massive concrete structures. Two scalar damage variables and a plastic-strain tensor were considered as internal variables, with intuitive evolution laws. The model was formulated on the basis of an effective stress tensor, afterwards split into tensile and compressive tensor components, each of them associated to an appropriate (and independent) scalar damage variable, with a particular evolution law. In a simple but elegant fashion, the stiffness degradation due to damage, as well as the stiffness recovering when passing from tension to compression (or backwards) was fully captured. An overall strain-driven formalism was adopted throughout in order to improve the algorithmic efficiency, as required for the analysis of large scale concrete structures (like dams), particularly under seismic conditions. Step-by-step algorithms were presented to illustrate code implementation.

Capability for simulating the concrete rate-sensitivity was also incorporated into the constitutive model, via slight modifications on the kinematics for the damage thresholds and with the addition of fluidity parameters and flow functions as in a classic Perzina 
regularization. The distinct concrete strain-rate sensibilities under tension or compression were easily attended, since independent regularizations were introduced in the respective evolution laws.

The model ability to predict concrete behaviour under inviscid or viscid conditions was checked in several situations, covering $1 \mathrm{D}, 2 \mathrm{D}$ and $3 \mathrm{D}$ applications, demonstrating its adequacy for the intended analyses. Among the possible outputs from the model, the structural distributions of both tensile and compressive damage variables provided helpful tools for the identification of the most affected concrete domains.

\section{REFERENCES}

1. J, F. Hall, The dynamic and earthquake behaviour of concrete dams: review of experimental behaviour and observational evidence. Soil Dyn. Earth. Eng. 7, No.2 (1988).

2. L. M. Kachanov, Time of rupture process under creep conditions. Izvestia Akademii Nauk, Otd Tech Nauk No.8, 26-31 (1958) (in Russian).

3. L. M. Kachanov, Introduction to Continuum Damage Mechanics, Martinus Nijhoff Publishers, Dordrecht, Netherlands (1986).

4. J. L. Chaboche, Continuum damage mechanics. Part I: General concepts. J. Appl. Mech., ASME 55, 59-64 (1988).

5. J. L. Chaboche, Continuum damage mechanics. Part II: Damage growth, crack initiation, and crack growth. J. Appl. Mech., ASME 55, 65-72 (1988).

6. J. Lemaitre, Coupled elasto-plasticity and damage constitutive equations. Comp. Meth. Appl. Mech. Eng. 51, 31-49 (1985).

7. J. Lemaitre, How to use damage mechanics. Nucl. Engng Des. 80, 233-245 (1984).

8. J. Lemaitre, A continuous damage mechanics model for ductile fracture. J. Eng. Mat. Tech., ASME 107, 83-89 (1985). 
9. D. Krajcinovic and G. U. Fonseka, The continuum damage theory of brittle materials. Part 1: General theory. J. Appl. Mech., ASME 48, 809-815 (1981).

10. G. U. Fonseka and D. Krajcinovic, The continuos damage theory of brittle materials. Part 2: Uniaxial and plane response modes. J. Appl. Mech., ASME 48, 816-824 (1981).

11. D. Krajcinovic, Constitutive equations for damaging materials. J. Appl. Mech., ASME 50, 355-360 (1983).

12. L. Resende and J. B. Martin, A progressive damage 'continuum' model for granular materials. Comp. Meth. Appl. Mech. Eng. 42, 1-18 (1984).

13. J. Lubliner, J. Oliver, S. Oller and E. Oñate, A plastic-damage model for concrete. Int. J. Solids Struct. 25, No.3, 299-326 (1989).

14. C. La Borderie, Y. Berthaud and G. Pijaudier-Cabot, Crack closure effects in continuum damage mechanics. Numerical implementation. Proc. 2nd Int. Conf. Comp. Aided Analysis Design Conc. Struct., Zell am See, Austria, 975-986 (1990).

15. J. Mazars and G. Pijaudier-Cabot, Continuum damage theory. Application to concrete. J. Eng. Mech., ASCE 115, No.2, 345-365 (1989).

16. J. Lemaitre and J. L. Chaboche, Aspects phénoménologiques de la rupture par endommagement. J. Méc. Appl. 2, No.3, 317-365 (1978).

17. J. C. Simo and J. W. Ju, Strain- and stress-based continuum damage models. I: Formulation. Int. J. Solids Struct. 23, No.7, 821-840 (1987).

18. J. Lubliner, On the thermodynamic foundations of non-linear solid mechanics. Int. J. Non-Linear Mech. 7, 237-254 (1972).

19. R. Faria and J. Oliver, A Rate Dependent Plastic-Damage Constitutive Model for Large Scale Computations in Concrete Structures, Monograph CIMNE, No.17, Barcelona, Spain (1993).

20. S. Murakami, Notion of continuum damage mechanics and its application to anisotropic creep damage theory. J. Eng. Mat. Techn., ASME 105, 99-105 (1983). 
21. J. W. Ju, Isotropic and anisotropic damage variables in continuum damage mechanics, J. Eng. Mech., ASCE 116, No.12, 2764-2770 (1990).

22. H. B. Kupfer, H. K. Hilsdorf and H. Rusch, Behaviour of concrete under biaxial stresses. J. Am. Conc. Inst. 66, No.8, 656-666 (1969).

23. J. Lubliner, Plasticity Theory, MacMillan Publishing Company, NY, USA (1990).

24. J. Oliver, M. Cervera, S. Oller and J. Lubliner, Isotropic damage models and smeared crack analysis of concrete. Proc. 2nd Int. Conf. Comp. Aided Analysis Design Conc. Struct., Zell am See, Austria, 945-957 (1990).

25. J. Oliver, A consistent characteristic length for smeared cracking models. Int. J. Num. Meth. Eng. 28, 461-474 (1989).

26. E. P. Sinha, K. H. Gerstle and L. G. Tulin, Stress-strain relations for concrete under cyclic loading. J. Am. Conc. Inst. 62, No.2, 195-210 (1964).

27. D. Z. Yankelevsky and H. W. Reinhardt, Model for cyclic compressive behaviour of concrete. J. Struct. Eng., ASCE 113, No.2, 228-240 (1987).

28. S. J. Green and S. R. Swanson, Static constitutive relations for concrete. AFWL-TR-72-244, US Air Force Weapons Laboratory (1973).

29. R. Faria, Seismic Behaviour of Concrete Dams Evaluated via a Continuum Damage Model, $\mathrm{PhD}$ Thesis, Oporto University (1994) (in Portuguese).

30. B. Bresler and A. C. Scordelis, Shear strength of reinforced concrete beams. J. Am. Conc. Inst. 60, No.1, 51-73 (1963).

31. W. Suaris, C. Ouyang and V. M. Fernando, Damage model for cyclic loading of concrete. J. Eng. Mech., ASCE 116, No.5, 1020-1035 (1990).

32. W. Suaris and S. P. Shah, Properties of concrete subjected to impact. J. Struct. Eng., ASCE 109, No.7, 1727-1741 (1983).

33. W. Suaris and S. P. Shah, Rate-sensitive damage theory for brittle solids. J. Eng. Mech., ASCE 110, No.6, 985-997 (1984). 
34. W. Suaris and S. P. Shah, Constitutive model for dynamic loading of concrete. $J$. Struct. Eng., ASCE 111, No.3, 563-576 (1985).

35. P. Chappuis, Modélisation Non-Linéaire du Comportement du Béton sous des Sollicitations Dynamiques, Doctoral Thesis No.155, Swiss Federal Institute of Technology (1987).

36. N. Bicanic and O. C. Zienkiewicz, Constitutive model for concrete under dynamic loading. Earth. Eng. Struct. Dyn. 11, 689-710 (1983).

37. M. Cervera, J. Oliver and O. Manzoli, A rate dependent isotropic damage model for the seismic analysis of concrete dams. Earth. Eng. Struct. Dyn. (to appear).

38. N. Bicanic and Pankaj, Some computational aspects of tensile strain localization modelling in concrete. Eng. Fract. Mech. 35, 697-708 (1990). 

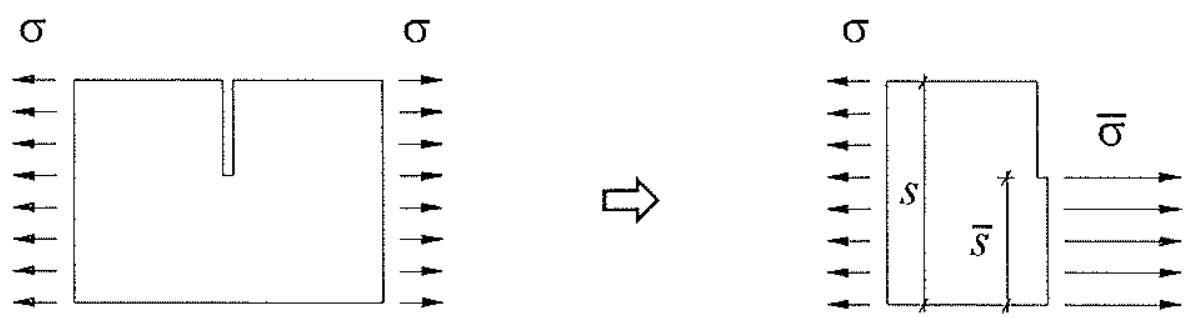

Fig. 1. Cauchy stress versus effective stress.

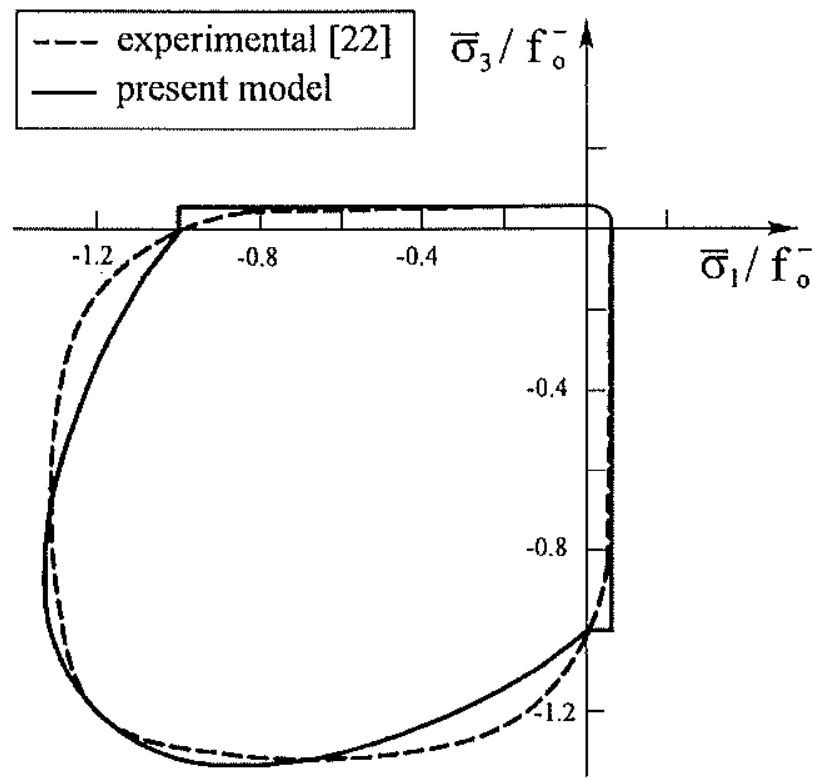

Fig. 2. Initial 2D elastic domain.

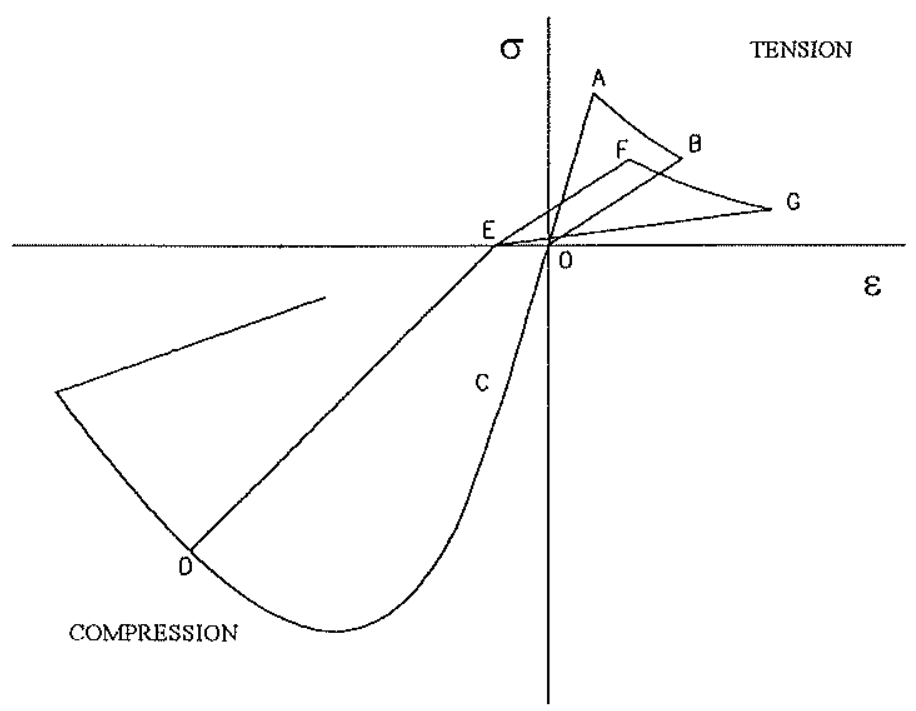

Fig. 3. Cyclic behaviour during a 1D test. 


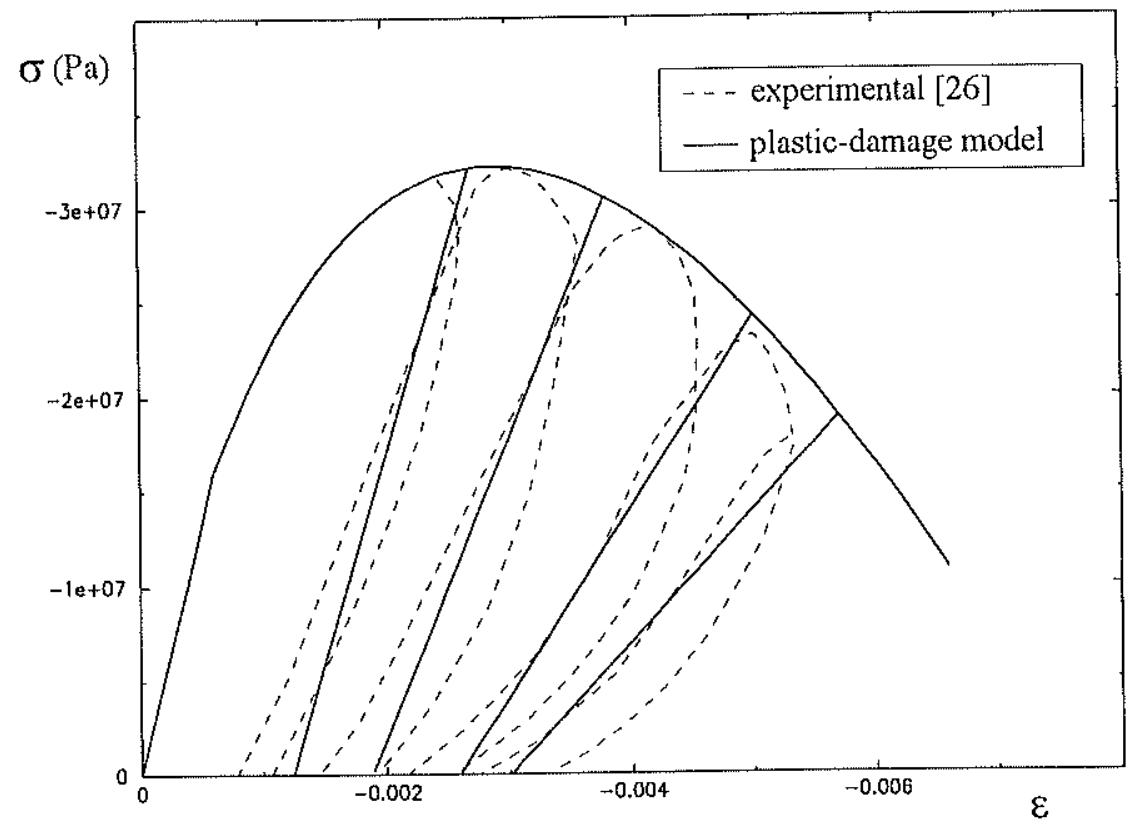

Fig. 4. 1D cyclic compressive test.

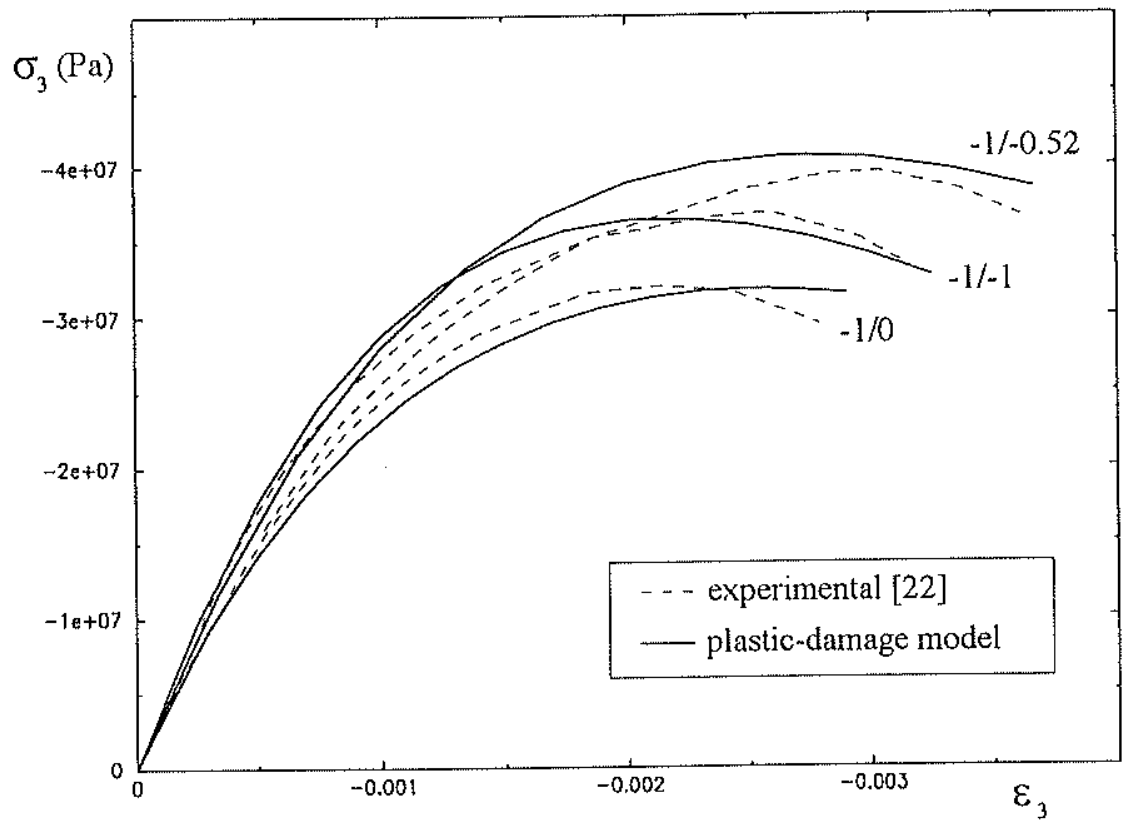

Fig. 5. 2D compressive test. 


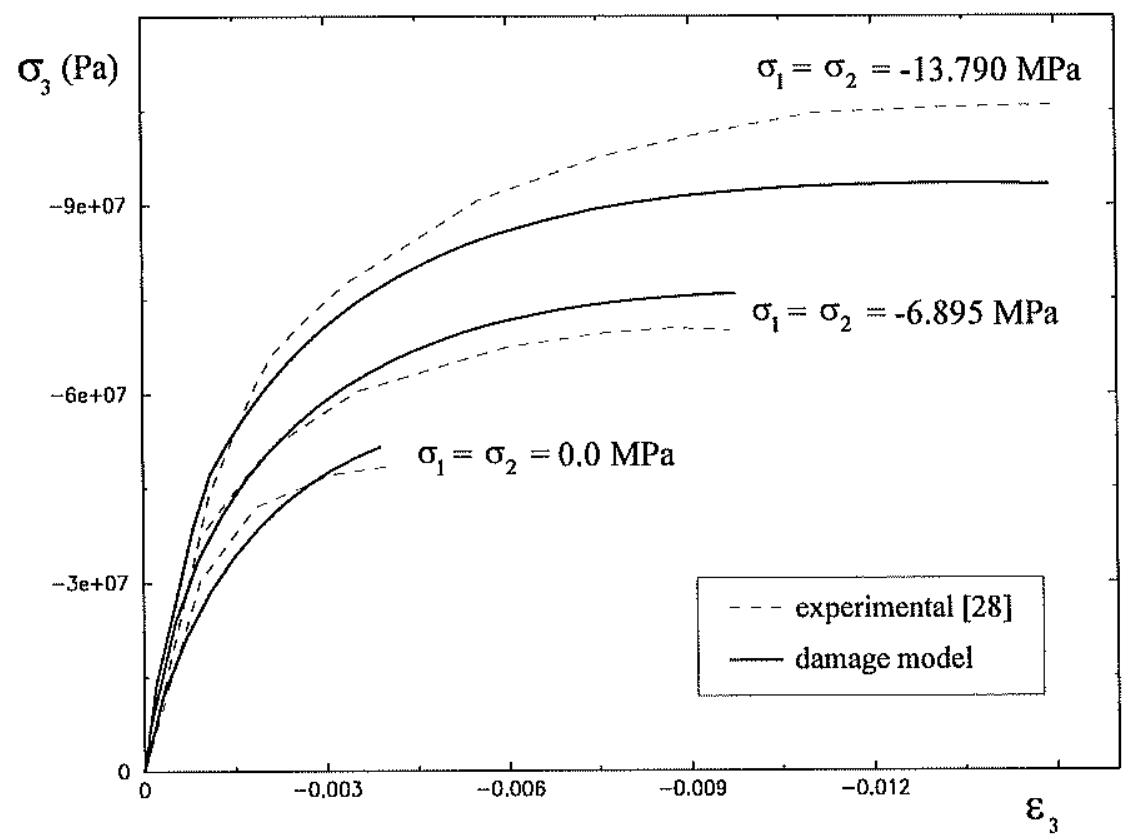

Fig. 6. 3D compressive test.

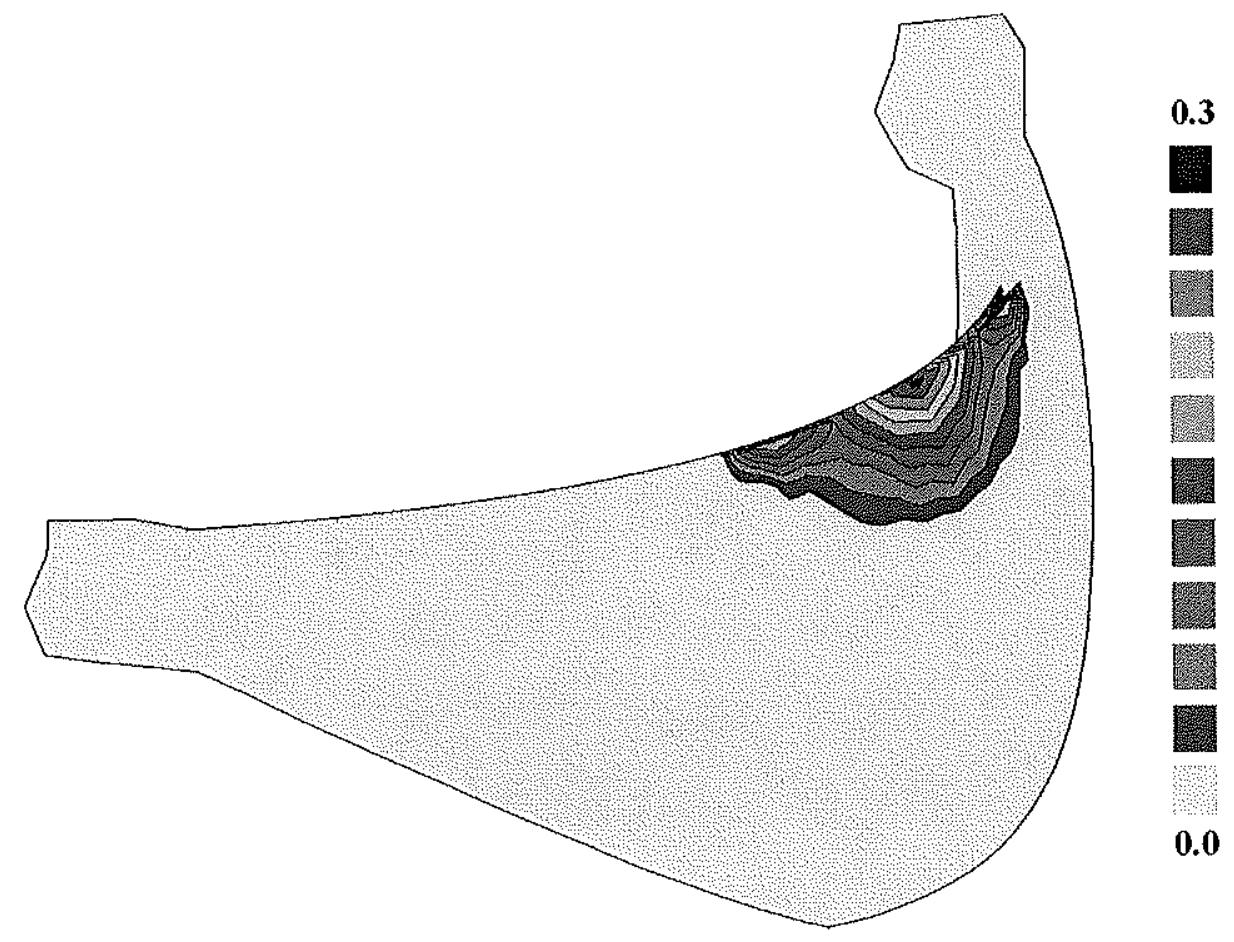

Fig. 7. Foz Côa dam: $d^{-}$distribution after a $1 \mathrm{~g}$ earthquake. 


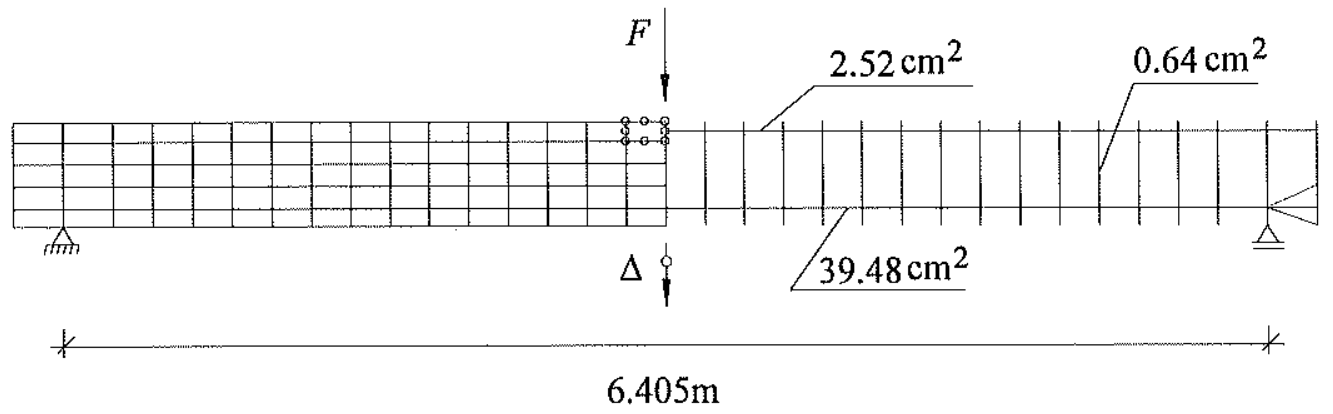

a) discretization (cross section: $0.307 \times 0.56 \mathrm{~m}^{2}$ )

b)
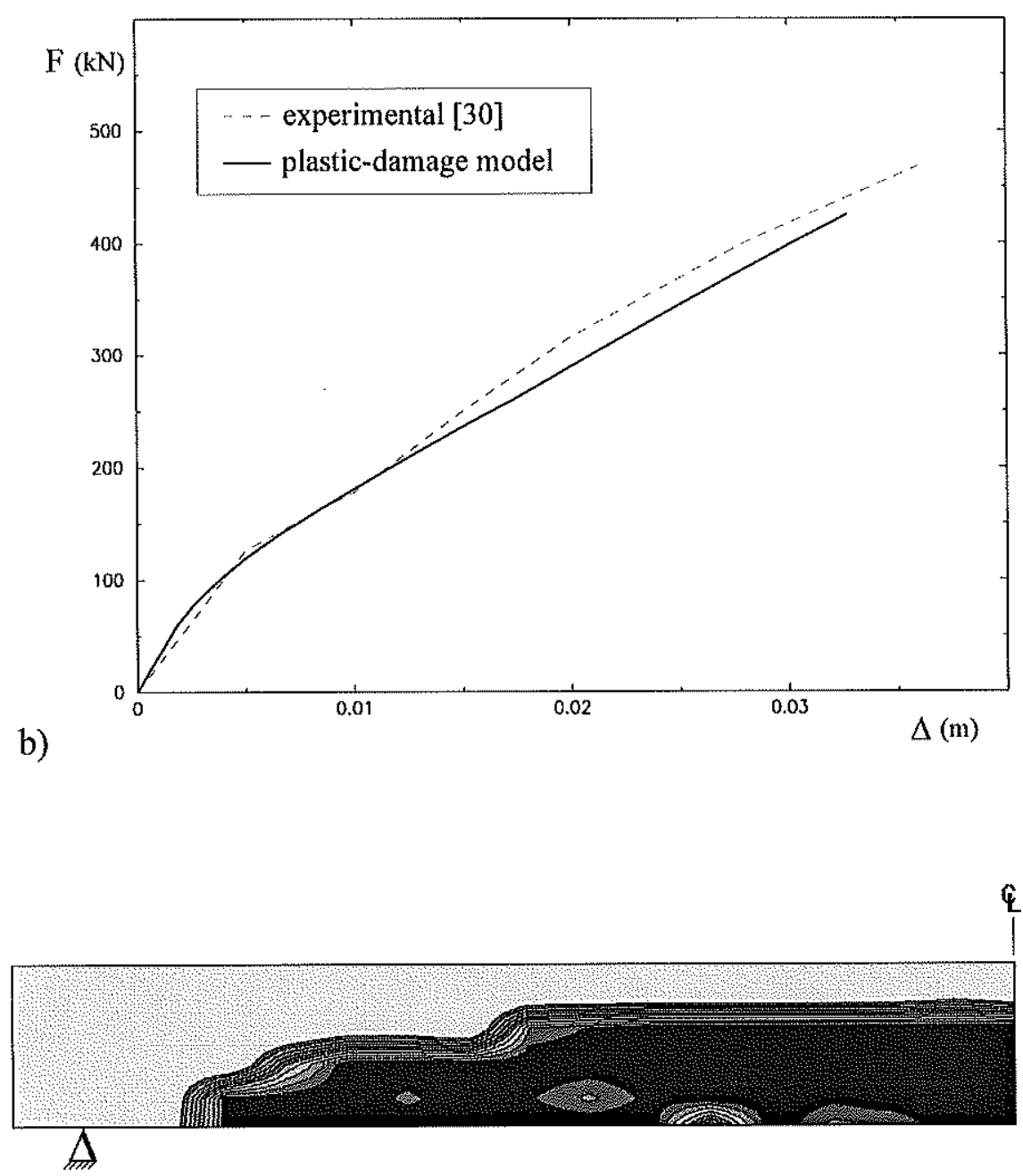

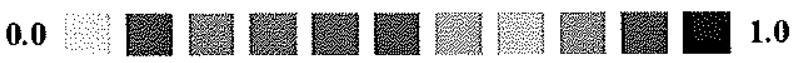

c) $d^{+}$distribution

Fig. 8. Beam A3 from [30]. 


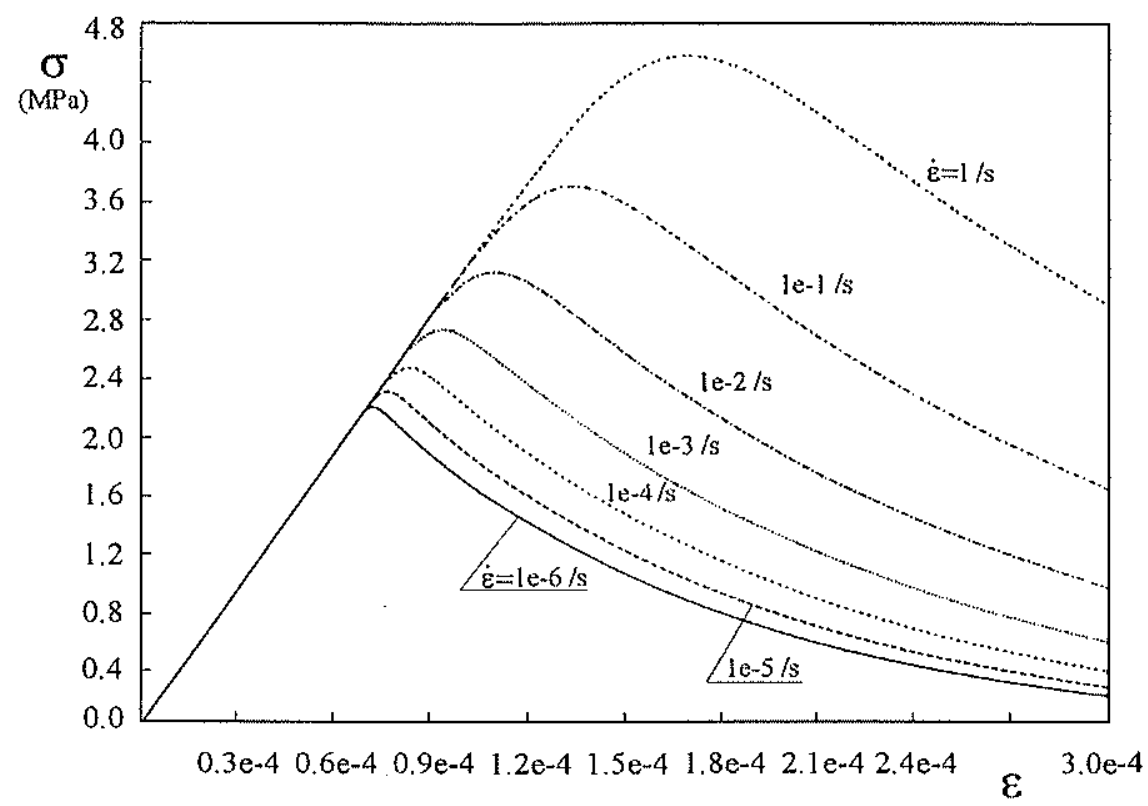

Fig. 9. Tensile strain-rate effect.

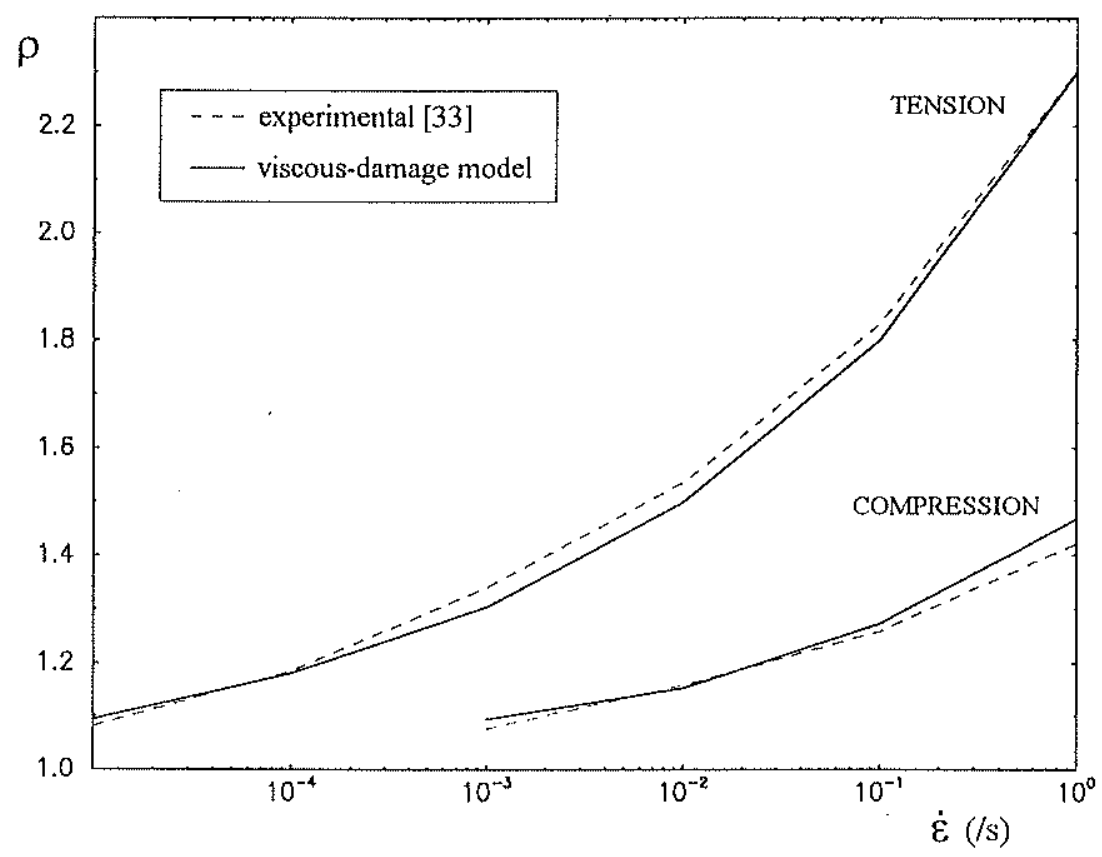

Fig. 10. Strain-rate dependency. 